nephron

Practice

\title{
UK Renal Registry 17th Annual Report: Chapter 1 UK Renal Replacement Therapy Incidence in 2013: National and Centre-specific Analyses
}

\author{
Julie Gilg ${ }^{a}$, Rishi Pruthi ${ }^{a}$, Damian Fogarty ${ }^{b}$ \\ ${ }^{a}$ UK Renal Registry, Bristol, UK; ${ }^{\mathrm{b}}$ Belfast Health and Social Care Trust
}

\begin{abstract}
Key Words
Acceptance rates - Clinical Commissioning Group - Comorbidity - Diabetes - Dialysis - End stage renal disease - End stage renal failure - Established renal failure - Glomerulonephritis - Haemodialysis - Incidence . Peritoneal dialysis . Registries - Renal replacement therapy - Transplantation . Treatment modality
\end{abstract}

\footnotetext{
Abstract

Introduction: This chapter describes the characteristics of adult patients starting renal replacement therapy (RRT) in the UK in 2013 and the incidence rates for RRT by Clinical Commissioning Groups and Health Boards (CCG/HBs) in the UK. Methods: Basic demographic and clinical characteristics, including presentation time (time between first being seen by a nephrologist and start of RRT), and age/gender standardised incidence ratios in $\mathrm{CCG} / \mathrm{HBs}$, are reported on patients starting RRT at all UK renal centres. Results: In 2013, RRT was started in 7,006 patients across the UK, with an incidence rate similar to 2012 at 109 per million
}

population (pmp). There were wide variations between $\mathrm{CCG} / \mathrm{HBs}$ in standardised incidence ratios. The median age for White patients was 66.0 and for non-White patients 57.0 years. Diabetic renal disease remained the single most common cause of renal failure (25\%). By 90 days, $66.1 \%$ of patients were on haemodialysis (HD), $19.0 \%$ on peritoneal dialysis (PD), 9.5\% had a functioning transplant and $5.3 \%$ had died or stopped treatment. There continued to be variability between centres in the use of PD as an initial treatment. The mean eGFR at the start of RRT was $8.5 \mathrm{ml} /$ $\min / 1.73 \mathrm{~m}^{2}$ similar to previous years. Late presentation ( $<90$ days) fell from $23.9 \%$ in 2006 to $18.4 \%$ in 2013 . Fiftyone percent of patients who started on HD had died within five years of starting. This compared to $33 \%$ and $5 \%$ for those starting on PD or transplant respectively. Conclusions: The incidence of new patients starting RRT in the UK has remained largely unchanged for almost 10 years in contrast to the rising numbers of prevalent patients $(+48 \%$ since 2003). The year on year increase in pre-emptive transplantation is encouraging but the variability between centres in the percentages starting on PD should be explored further. 


\section{Introduction}

This chapter contains analyses of adult patients starting renal replacement therapy (RRT) in the UK in 2013. The methodology and results for these analyses are in three separate sections: geographical variations in incidence rates, the demographic and clinical characteristics of patients starting RRT and analyses of late presentation and delayed referral.

\section{Definitions}

The definition of incident patients is given in detail in appendix B: Definitions and Analysis Criteria (www. renalreg.org). In brief, it is all patients over 18 who commenced RRT in the UK in 2013 and who did not recover renal function within 90 days. Note that this does not include those with a failed renal transplant who returned to dialysis.

Differences may be seen in the 2008 to 2012 numbers now quoted when compared with previous publications because of retrospective updating of data in collaboration with renal centres, in particular for patients who were initially thought to have acute renal failure. Where applicable and possible, pre-emptive transplant patients were allocated to their work up centre rather than their transplant centre. However, this was not possible for all such patients and consequently some patients probably remain incorrectly allocated to the transplanting centre. The term established renal failure (ERF) as used within this chapter is synonymous with the terms end stage renal failure/disease (ESRF or ESRD).

\section{UK Renal Registry coverage}

The UK Renal Registry (UKRR) received individual patient level data from all 71 adult renal centres in the UK (five renal centres in Wales, five in Northern Ireland, nine in Scotland, 52 in England). Data from centres in Scotland were obtained from the Scottish Renal Registry. Data on children and young adults can be found in chapter 4: Demography of the UK Paediatric Renal Replacement Therapy population in 2013.

\section{Renal Association Guidelines}

Table 1.1 lists the relevant items from the Renal Association Guidelines on the Planning, Initiating and

Table 1.1. Summary of Renal Association audit measures relevant to RRT incidence

\begin{tabular}{|c|c|c|}
\hline RA audit measure & Reported & Reason for non-inclusion/comment \\
\hline $\begin{array}{l}\text { Percentage of patients commencing RRT referred }<3 \text { months } \\
\text { and }<12 \text { months before date of starting RRT }\end{array}$ & Yes & $\begin{array}{l}\text { Registry dataset allows reporting on time elapsed between } \\
\text { date first seen and start of RRT }\end{array}$ \\
\hline $\begin{array}{l}\text { Percentage of incident RRT patients followed up for }>3 \\
\text { months in dedicated pre-dialysis or low clearance clinic }\end{array}$ & No & Not in UKRR dataset \\
\hline $\begin{array}{l}\text { Proportion of incident patients on UK transplant waiting list at } \\
\text { RRT initiation }\end{array}$ & No & Not in UKRR dataset \\
\hline $\begin{array}{l}\text { Proportion of incident RRT patients transplanted pre-emptively } \\
\text { from living donors and cadaveric donors }\end{array}$ & Yes & \\
\hline Mean eGFR at time of pre-emptive transplantation & No & $\begin{array}{l}\text { Numbers with data will be small, the UKRR will consider } \\
\text { doing a combined years analysis in future reports }\end{array}$ \\
\hline $\begin{array}{l}\text { Proportion of incident patients commencing peritoneal or } \\
\text { home haemodialysis }\end{array}$ & Part & Proportion starting on PD is reported \\
\hline $\begin{array}{l}\text { Proportion of patients who have undergone a formal education } \\
\text { programme prior to initiation of RRT }\end{array}$ & No & Not in UKRR dataset \\
\hline $\begin{array}{l}\text { Proportion of haemodialysis patients who report that they have } \\
\text { been offered a choice of RRT modality }\end{array}$ & No & Not in UKRR dataset \\
\hline $\begin{array}{l}\text { Proportion of patients who have initiated dialysis in an unplanned } \\
\text { fashion who have undergone formal education by } 3 \text { months. }\end{array}$ & No & Not in UKRR dataset \\
\hline $\begin{array}{l}\text { Evidence of formal continuing education programme for } \\
\text { patients on dialysis }\end{array}$ & No & Not in UKRR dataset \\
\hline $\begin{array}{l}\text { Proportion of incident patients known to nephrology services } \\
\text { for } 3 \text { months or more prior to initiation (planned initiation) }\end{array}$ & No & Not in UKRR dataset \\
\hline $\begin{array}{l}\text { Proportion of planned initiations with established access or } \\
\text { pre-emptive transplantation }\end{array}$ & Yes & $\begin{array}{l}\text { See appendix } \mathrm{F} \text { for pre-emptive transplantation, and see } \\
\text { chapter } 10 \text { for dialysis access }\end{array}$ \\
\hline Inpatient/outpatient status of planned initiations & No & Not in UKRR dataset \\
\hline Mean eGFR at start of renal replacement therapy & Part & $\begin{array}{l}\text { Reported but not at centre level due to poor data } \\
\text { completness }\end{array}$ \\
\hline
\end{tabular}


Table 1.2. Number of new adult patients starting RRT in the UK in 2013

\begin{tabular}{lccccc}
\hline & England & N Ireland & Scotland & Wales & UK \\
\hline Number starting RRT & 5,964 & 180 & 502 & 360 & $\mathbf{7 , 0 0 6}$ \\
Total estimated population mid-2013 (millions) & 53.9 & 1.8 & 5.3 & 3.1 & $\mathbf{6 4 . 1}$ \\
Incidence rate (pmp) & 111 & 98 & 94 & 117 & $\mathbf{1 0 9}$ \\
$(95 \%$ CI) & $(108-114)$ & $(84-113)$ & $(86-102)$ & $(105-129)$ & $(\mathbf{1 0 7}-\mathbf{1 1 2})$ \\
\hline
\end{tabular}

* Data from the Office for National Statistics, National Records of Scotland and the Northern Ireland Statistics and Research Agency - based on the 2011 census

Withdrawal of Renal Replacement Therapy [1]. Many of the audit measures are not currently reported by the UKRR; mainly due to a high proportion of incomplete data or because the relevant data item(s) is not currently within the specified UKRR dataset. Over time we hope to work with the renal community to improve reporting across the range of these measures.

\section{Geographical variation in incidence rates}

\section{Introduction}

Over the years, there have been wide variations in incidence rates between renal centres. Equity of access to RRT is an important aim but hard to assess as the need for RRT depends on many variables including medical, social and demographic factors such as underlying conditions, age, gender, social deprivation and ethnicity. Thus, comparison of crude incidence rates by geographical area can be misleading. This year's report again uses age and gender standardisation of Clinical Commissioning Group/Health Board (CCG/HB) rates as well as showing crude rates. It also gives the ethnic minority percentage of each area as this influences incidence rates.

\section{Methods}

CCG/HB level

Crude incidence rates per million population (pmp) and age/ gender standardised incidence ratios were calculated as detailed in appendix D: Methodology used for Analyses (www.renalreg. org).

\section{Centre level}

For the methodology used to estimate catchment populations see appendix E: Methodology for Estimating Catchment Populations (www.renalreg.org).

\section{Results}

\section{Overall}

In 2013, the number of adult patients starting RRT in the UK was 7,006 equating to an incidence rate of 109 pmp (table 1.2), compared with 108 pmp in 2012. Wales remained the country with the highest incidence rate (figure 1.1). For England, incidence rates have been stable for the last eight years. There continued to be very marked gender differences in incidence rates which were $141 \mathrm{pmp}$ (95\% CI 137-145) in males and 79 pmp (95\% CI 76-82) in females.

The denominators used for these rates were the entire population i.e. they include under 18 year olds. When incident patients aged under 18 were included in the numerator the UK rate was $111 \mathrm{pmp}$.

\section{CCG/HB level}

Table 1.3 shows incidence rates and standardised incidence ratios for CCG/HBs. There were wide variations

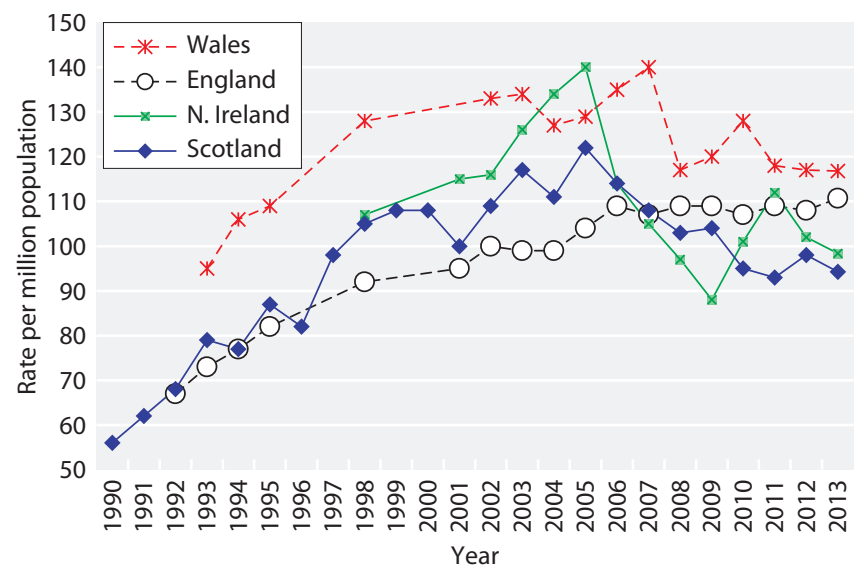

Fig. 1.1. RRT incidence rates in the countries of the UK 19902013 
Table 1.3. Crude adult incidence rates (pmp) and age/gender standardised incidence ratios 2008-2013

CCG/HB - CCG in England, Health and Social Care Areas in Northern Ireland, Local Health Boards in Wales and Health Boards in Scotland $\mathrm{O} / \mathrm{E}$ - standardised incidence ratio

LCL - lower $95 \%$ confidence limit

UCL - upper 95\% confidence limit

pmp - per million population

* - per year

Areas with significantly low incidence ratios over six years are italicised in greyed areas, those with significantly high incidence ratios over six years are bold in greyed areas. For the full methodology see appendix D

Confidence intervals are not given for the crude rates per million population but figures D1 and D2 in appendix D can be used to determine if a CCG/HB falls within the $95 \%$ confidence interval around the national average rate

Mid-2012 population data from the Office for National Statistics, National Records of Scotland and the Northern Ireland Statistics and Research Agency - based on the 2011 census

$\%$ non-White - percentage of the CCG/HB population that is non-White, from 2011 census

\begin{tabular}{|c|c|c|c|c|c|c|c|c|c|c|c|c|c|c|}
\hline UK Area & CCG/HB & $\begin{array}{l}\text { Tot pop } \\
(2012)\end{array}$ & $\begin{array}{c}2008 \\
\mathrm{O} / \mathrm{E}\end{array}$ & $\begin{array}{r}2009 \\
\mathrm{O} / \mathrm{E}\end{array}$ & $\begin{array}{r}2010 \\
\mathrm{O} / \mathrm{E}\end{array}$ & $\begin{array}{l}2011 \\
\mathrm{O} / \mathrm{E}\end{array}$ & $\begin{array}{l}2012 \\
\mathrm{O} / \mathrm{E}\end{array}$ & $\mathrm{O} / \mathrm{E}$ & $\begin{array}{l}3 \\
\text { Crude } \\
\text { rate } \\
\text { pmp }\end{array}$ & $\mathrm{O} / \mathrm{E}$ & 2008 & UCL & $\begin{array}{c}\text { Crude } \\
\text { rate } \\
\text { pmp }^{*}\end{array}$ & $\begin{array}{c}\% \\
\text { non- } \\
\text { White }\end{array}$ \\
\hline \multirow{6}{*}{$\begin{array}{l}\text { Cheshire, } \\
\text { Warrington } \\
\text { and Wirral }\end{array}$} & NHS Eastern Cheshire & 195,300 & 0.51 & 0.75 & 0.85 & 0.74 & 0.74 & 0.68 & 87 & 0.71 & 0.59 & 0.86 & 88 & 3.7 \\
\hline & NHS South Cheshire & 176,800 & 0.61 & 0.70 & 0.71 & 0.74 & 0.59 & 1.15 & 136 & 0.75 & 0.61 & 0.92 & 86 & 2.9 \\
\hline & NHS Vale Royal & 102,100 & 0.54 & 0.88 & 0.81 & 0.87 & 0.78 & 1.27 & 147 & 0.86 & 0.67 & 1.11 & 96 & 2.1 \\
\hline & NHS Warrington & 203,700 & 0.61 & 1.01 & 0.61 & 0.46 & 0.86 & 0.67 & 74 & 0.70 & 0.57 & 0.86 & 75 & 4.1 \\
\hline & NHS West Cheshire & 228,100 & 0.61 & 0.90 & 1.18 & 1.04 & 0.81 & 1.01 & 123 & 0.92 & 0.79 & 1.08 & 109 & 2.8 \\
\hline & NHS Wirral & 320,200 & 0.72 & 0.81 & 0.88 & 0.94 & 0.59 & 0.99 & 119 & 0.82 & 0.71 & 0.95 & 95 & 3.0 \\
\hline \multirow{5}{*}{$\begin{array}{l}\text { Durham, } \\
\text { Darlington }\end{array}$} & NHS Darlington & 105,200 & 1.05 & 0.95 & 0.97 & 0.94 & 1.27 & 0.83 & 95 & 1.00 & 0.79 & 1.26 & 111 & 3.8 \\
\hline & $\begin{array}{l}\text { NHS Durham Dales, Easington and } \\
\text { Sedgefield }\end{array}$ & 273,000 & 0.71 & 0.98 & 1.03 & 1.09 & 0.84 & 1.00 & 121 & 0.94 & 0.81 & 1.09 & 110 & 1.2 \\
\hline & NHS Hartlepool and Stockton-on-Tees & 284,600 & 1.01 & 0.69 & 0.81 & 0.91 & 1.04 & 0.86 & 95 & 0.89 & 0.76 & 1.03 & 95 & 4.4 \\
\hline & NHS North Durham & 241,300 & 0.68 & 0.52 & 0.49 & 0.55 & 1.28 & 0.64 & 75 & 0.69 & 0.58 & 0.84 & 78 & 2.5 \\
\hline & NHS South Tees & 273,700 & 1.03 & 0.77 & 1.06 & 0.93 & 0.96 & 1.20 & 135 & 0.99 & 0.86 & 1.15 & 108 & 6.7 \\
\hline \multirow{12}{*}{$\begin{array}{l}\text { Greater } \\
\text { Manchester }\end{array}$} & NHS Bolton & 279,000 & 0.89 & 0.84 & 1.40 & 0.94 & 0.90 & 0.82 & 86 & 0.96 & 0.83 & 1.12 & 99 & 18.1 \\
\hline & NHS Bury & 186,200 & 0.78 & 0.82 & 0.73 & 0.71 & 1.35 & 0.79 & 86 & 0.86 & 0.71 & 1.05 & 91 & 10.8 \\
\hline & NHS Central Manchester & 182,400 & 2.20 & 1.79 & 2.08 & 1.11 & 1.70 & 2.22 & 154 & 1.85 & 1.56 & 2.18 & 125 & 48.0 \\
\hline & NHS Heywood, Middleton \& Rochdale & 212,000 & 1.01 & 1.14 & 0.82 & 1.22 & 1.26 & 1.10 & 113 & 1.09 & 0.92 & 1.29 & 109 & 18.3 \\
\hline & NHS North Manchester & 167,100 & 0.99 & 1.68 & 0.93 & 1.50 & 1.43 & 1.48 & 120 & 1.34 & 1.11 & 1.62 & 106 & 30.8 \\
\hline & NHS Oldham & 225,900 & 1.15 & 0.86 & 0.88 & 1.03 & 0.71 & 0.96 & 97 & 0.93 & 0.78 & 1.11 & 91 & 22.5 \\
\hline & NHS Salford & 237,100 & 1.07 & 0.97 & 1.39 & 0.74 & 0.87 & 1.11 & 110 & 1.02 & 0.87 & 1.21 & 98 & 9.9 \\
\hline & NHS South Manchester & 161,300 & 0.90 & 0.89 & 0.99 & 1.17 & 1.18 & 1.23 & 105 & 1.06 & 0.86 & 1.31 & 89 & 19.6 \\
\hline & NHS Stockport & 283,900 & 0.80 & 0.53 & 0.92 & 0.87 & 0.64 & 0.51 & 60 & 0.71 & 0.60 & 0.84 & 80 & 7.9 \\
\hline & NHS Tameside and Glossop & 253,400 & 0.69 & 0.86 & 0.96 & 0.97 & 0.59 & 1.12 & 122 & 0.87 & 0.73 & 1.02 & 91 & 8.2 \\
\hline & NHS Trafford & 228,500 & 0.55 & 1.08 & 1.28 & 0.54 & 1.15 & 1.13 & 123 & 0.96 & 0.81 & 1.13 & 101 & 14.5 \\
\hline & NHS Wigan Borough & 318,700 & 0.79 & 0.58 & 0.77 & 1.04 & 0.77 & 0.73 & 82 & 0.78 & 0.67 & 0.91 & 85 & 2.7 \\
\hline \multirow[t]{8}{*}{ Lancashire } & NHS Blackburn with Darwen & 147,700 & 0.52 & 0.88 & 1.04 & 1.44 & 1.22 & 0.91 & 88 & 1.00 & 0.81 & 1.24 & 94 & 30.8 \\
\hline & NHS Blackpool & 142,000 & 1.00 & 0.98 & 0.62 & 0.85 & 1.45 & 1.12 & 134 & 1.01 & 0.83 & 1.22 & 116 & 3.3 \\
\hline & NHS Chorley and South Ribble & 167,900 & 0.83 & 1.30 & 0.55 & 1.02 & 0.75 & 1.31 & 149 & 0.96 & 0.80 & 1.16 & 106 & 2.9 \\
\hline & NHS East Lancashire & 371,600 & 0.71 & 0.85 & 0.74 & 0.92 & 0.54 & 0.89 & 100 & 0.78 & 0.67 & 0.90 & 84 & 11.9 \\
\hline & NHS Fylde \& Wyre & 165,000 & 0.70 & 0.86 & 0.69 & 0.54 & 0.76 & 0.79 & 109 & 0.73 & 0.59 & 0.89 & 97 & 2.1 \\
\hline & NHS Greater Preston & 202,000 & 0.88 & 0.67 & 0.54 & 0.52 & 1.00 & 0.84 & 89 & 0.74 & 0.61 & 0.91 & 77 & 14.7 \\
\hline & NHS Lancashire North & 158,500 & 0.34 & 0.62 & 0.57 & 1.00 & 0.66 & 0.60 & 69 & 0.63 & 0.50 & 0.80 & 71 & 4.0 \\
\hline & NHS West Lancashire & 110,900 & 1.03 & 0.62 & 0.63 & 0.84 & 0.76 & 0.67 & 81 & 0.76 & 0.59 & 0.98 & 89 & 1.9 \\
\hline \multirow[t]{6}{*}{ Merseyside } & NHS Halton & 125,700 & 0.31 & 1.07 & 0.86 & 1.59 & 0.97 & 0.95 & 103 & 0.96 & 0.77 & 1.20 & 101 & 2.2 \\
\hline & NHS Knowsley & 145,900 & 0.46 & 0.78 & 0.93 & 1.16 & 1.28 & 0.69 & 75 & 0.89 & 0.71 & 1.10 & 94 & 2.8 \\
\hline & NHS Liverpool & 469,700 & 1.16 & 1.19 & 0.87 & 1.08 & 1.19 & 0.98 & 98 & 1.08 & 0.96 & 1.21 & 105 & 11.1 \\
\hline & NHS South Sefton & 159,400 & 1.12 & 0.77 & 1.28 & 1.36 & 1.02 & 1.27 & 151 & 1.14 & 0.95 & 1.35 & 131 & 2.2 \\
\hline & NHS Southport and Formby & 114,300 & 0.55 & 0.80 & 0.61 & 0.93 & 0.73 & 1.36 & 184 & 0.84 & 0.67 & 1.05 & 109 & 3.1 \\
\hline & NHS St Helens & 176,100 & 0.76 & 0.70 & 0.92 & 0.74 & 0.88 & 0.63 & 74 & 0.77 & 0.63 & 0.94 & 88 & 2.0 \\
\hline
\end{tabular}


Table 1.3. Continued

\begin{tabular}{|c|c|c|c|c|c|c|c|c|c|c|c|c|c|c|}
\hline UK Area & CCG/HB & $\begin{array}{l}\text { Tot pop } \\
(2012)\end{array}$ & $\begin{array}{c}2008 \\
\mathrm{O} / \mathrm{E}\end{array}$ & $\begin{array}{c}2009 \\
\mathrm{O} / \mathrm{E}\end{array}$ & $\begin{array}{c}2010 \\
\mathrm{O} / \mathrm{E}\end{array}$ & $\begin{array}{l}2011 \\
\mathrm{O} / \mathrm{E}\end{array}$ & $\begin{array}{c}2012 \\
\mathrm{O} / \mathrm{E}\end{array}$ & $\mathrm{O} / \mathrm{E}$ & $\begin{array}{l}\text { Crude } \\
\text { rate } \\
\text { pmp }\end{array}$ & $\mathrm{O} / \mathrm{E}$ & $2008-$ & UCL & $\begin{array}{c}\text { Crude } \\
\text { rate } \\
\text { pmp }^{*}\end{array}$ & $\begin{array}{c}\% \\
\text { non- } \\
\text { White }\end{array}$ \\
\hline \multirow{8}{*}{$\begin{array}{l}\text { Cumbria, } \\
\text { Northum- } \\
\text { berland, } \\
\text { Tyne and } \\
\text { Wear }\end{array}$} & NHS Cumbria & 505,200 & 0.70 & 0.61 & 0.73 & 0.59 & 0.61 & 0.89 & 115 & 0.69 & 0.61 & 0.78 & 86 & 1.5 \\
\hline & NHS Gateshead & 200,200 & 0.55 & 0.89 & 0.78 & 0.75 & 0.88 & 0.48 & 55 & 0.72 & 0.59 & 0.88 & 81 & 3.7 \\
\hline & NHS Newcastle North and East & 141,600 & 0.96 & 1.03 & 0.88 & 0.85 & 0.70 & 0.46 & 42 & 0.81 & 0.63 & 1.04 & 73 & 10.7 \\
\hline & NHS Newcastle West & 140,900 & 1.25 & 0.87 & 0.67 & 0.86 & 0.86 & 0.91 & 92 & 0.90 & 0.72 & 1.13 & 89 & 18.3 \\
\hline & NHS North Tyneside & 201,400 & 0.50 & 0.89 & 0.90 & 0.62 & 0.87 & 0.94 & 109 & 0.79 & 0.65 & 0.95 & 89 & 3.4 \\
\hline & NHS Northumberland & 316,100 & 0.68 & 0.62 & 0.60 & 0.82 & 0.78 & 0.62 & 79 & 0.69 & 0.59 & 0.80 & 85 & 1.6 \\
\hline & NHS South Tyneside & 148,400 & 0.55 & 1.31 & 0.73 & 1.06 & 0.59 & 0.75 & 88 & 0.83 & 0.67 & 1.03 & 94 & 4.1 \\
\hline & NHS Sunderland & 275,700 & 0.87 & 0.95 & 1.04 & 0.75 & 0.87 & 0.57 & 65 & 0.84 & 0.72 & 0.98 & 93 & 4.1 \\
\hline \multirow{8}{*}{$\begin{array}{l}\text { North } \\
\text { Yorkshire } \\
\text { and } \\
\text { Humber }\end{array}$} & NHS East Riding of Yorkshire & 314,500 & 1.00 & 0.93 & 0.69 & 0.72 & 0.74 & 0.48 & 64 & 0.76 & 0.65 & 0.88 & 96 & 1.9 \\
\hline & $\begin{array}{l}\text { NHS Hambleton, Richmondshire and } \\
\text { Whitby }\end{array}$ & 153,400 & 0.60 & 0.90 & 0.76 & 0.68 & 1.25 & 0.92 & 117 & 0.85 & 0.70 & 1.04 & 105 & 2.7 \\
\hline & NHS Harrogate and Rural District & 158,600 & 0.65 & 1.01 & 0.65 & 0.95 & 0.89 & 0.52 & 63 & 0.78 & 0.63 & 0.96 & 92 & 3.7 \\
\hline & NHS Hull & 257,200 & 1.06 & 1.00 & 0.94 & 0.75 & 0.79 & 0.93 & 93 & 0.91 & 0.77 & 1.08 & 89 & 5.9 \\
\hline & NHS North East Lincolnshire & 159,700 & 1.09 & 0.85 & 0.69 & 1.34 & 0.67 & 0.82 & 94 & 0.91 & 0.74 & 1.11 & 101 & 2.6 \\
\hline & NHS North Lincolnshire & 168,400 & 0.90 & 0.73 & 0.69 & 1.50 & 1.13 & 1.06 & 125 & 1.00 & 0.84 & 1.20 & 115 & 4.0 \\
\hline & NHS Scarborough and Ryedale & 110,500 & 0.80 & 0.92 & 0.58 & 0.56 & 0.91 & 0.69 & 91 & 0.74 & 0.58 & 0.95 & 95 & 2.5 \\
\hline & NHS Vale of York & 346,100 & 0.74 & 0.65 & 0.69 & 1.08 & 0.92 & 0.78 & 90 & 0.81 & 0.70 & 0.93 & 91 & 4.0 \\
\hline \multirow{5}{*}{$\begin{array}{l}\text { South } \\
\text { Yorkshire } \\
\text { and } \\
\text { Bassetlaw }\end{array}$} & NHS Barnsley & 233,700 & 1.10 & 0.93 & 1.18 & 0.80 & 1.03 & 1.01 & 116 & 1.01 & 0.86 & 1.18 & 112 & 2.1 \\
\hline & NHS Bassetlaw & 113,200 & 0.61 & 0.68 & 0.92 & 0.82 & 1.04 & 1.23 & 150 & 0.89 & 0.70 & 1.12 & 105 & 2.6 \\
\hline & NHS Doncaster & 302,700 & 0.80 & 1.03 & 0.93 & 1.05 & 0.81 & 1.14 & 129 & 0.96 & 0.83 & 1.11 & 105 & 4.7 \\
\hline & NHS Rotherham & 258,400 & 1.39 & 0.95 & 1.11 & 0.69 & 0.83 & 0.78 & 89 & 0.95 & 0.82 & 1.11 & 106 & 6.4 \\
\hline & NHS Sheffield & 557,400 & 1.14 & 1.29 & 1.05 & 1.00 & 1.23 & 0.97 & 99 & 1.11 & 1.00 & 1.23 & 111 & 16.3 \\
\hline \multirow{10}{*}{$\begin{array}{l}\text { West } \\
\text { Yorkshire }\end{array}$} & NHS Airedale, Wharfedale and Craven & 158,200 & 0.56 & 1.03 & 0.56 & 0.49 & 0.64 & 0.84 & 101 & 0.69 & 0.55 & 0.86 & 80 & 11.1 \\
\hline & NHS Bradford City & 82,300 & 2.10 & 0.38 & 3.32 & 1.87 & 2.66 & 2.60 & 170 & 2.15 & 1.70 & 2.73 & 138 & 72.2 \\
\hline & NHS Bradford Districts & 333,500 & 1.26 & 0.96 & 1.21 & 1.08 & 1.35 & 1.05 & 102 & 1.15 & 1.01 & 1.31 & 108 & 28.7 \\
\hline & NHS Calderdale & 205,300 & 0.89 & 0.97 & 0.52 & 0.59 & 0.77 & 1.06 & 117 & 0.80 & 0.66 & 0.97 & 85 & 10.3 \\
\hline & NHS Greater Huddersfield & 238,800 & 0.61 & 0.76 & 0.82 & 0.91 & 1.10 & 0.89 & 96 & 0.85 & 0.71 & 1.01 & 89 & 17.4 \\
\hline & NHS Leeds North & 199,600 & 1.40 & 0.73 & 0.65 & 0.81 & 0.77 & 0.84 & 95 & 0.86 & 0.72 & 1.04 & 95 & 17.4 \\
\hline & NHS Leeds South and East & 238,300 & 1.13 & 0.67 & 0.73 & 0.97 & 0.75 & 0.95 & 92 & 0.86 & 0.72 & 1.03 & 82 & 18.3 \\
\hline & NHS Leeds West & 319,800 & 0.69 & 0.96 & 0.59 & 0.64 & 0.71 & 1.13 & 106 & 0.79 & 0.67 & 0.93 & 72 & 10.8 \\
\hline & NHS North Kirklees & 186,700 & 0.94 & 1.47 & 1.06 & 1.24 & 0.54 & 1.48 & 150 & 1.12 & 0.94 & 1.34 & 111 & 25.3 \\
\hline & NHS Wakefield & 327,600 & 0.76 & 0.58 & 0.88 & 0.91 & 1.07 & 0.86 & 98 & 0.84 & 0.73 & 0.98 & 93 & 4.6 \\
\hline \multirow{7}{*}{$\begin{array}{l}\text { Arden, } \\
\text { Hereford- } \\
\text { shire and } \\
\text { Worcester- } \\
\text { shire }\end{array}$} & NHS Coventry and Rugby & 423,900 & 1.32 & 1.62 & 1.33 & 1.43 & 1.74 & 1.26 & 127 & 1.45 & 1.31 & 1.61 & 142 & 22.2 \\
\hline & NHS Herefordshire & 184,900 & 0.93 & 1.13 & 0.71 & 0.82 & 0.90 & 0.76 & 97 & 0.87 & 0.73 & 1.04 & 109 & 1.8 \\
\hline & NHS Redditch and Bromsgrove & 178,700 & 1.17 & 1.30 & 0.97 & 0.79 & 1.23 & 0.67 & 78 & 1.02 & 0.85 & 1.22 & 115 & 6.0 \\
\hline & NHS South Warwickshire & 259,200 & 0.88 & 0.79 & 0.74 & 1.01 & 0.65 & 0.57 & 69 & 0.77 & 0.66 & 0.91 & 91 & 7.0 \\
\hline & NHS South Worcestershire & 292,300 & 0.82 & 0.86 & 0.70 & 0.71 & 0.84 & 0.75 & 92 & 0.78 & 0.67 & 0.91 & 94 & 3.7 \\
\hline & NHS Warwickshire North & 188,000 & 1.31 & 0.96 & 1.61 & 1.09 & 0.80 & 0.69 & 80 & 1.07 & 0.90 & 1.27 & 120 & 6.5 \\
\hline & NHS Wyre Forest & 98,100 & 1.01 & 1.24 & 0.93 & 1.06 & 0.89 & 0.64 & 82 & 0.96 & 0.76 & 1.21 & 119 & 2.8 \\
\hline \multirow{7}{*}{$\begin{array}{l}\text { Birmingham } \\
\text { and the } \\
\text { Black } \\
\text { Country }\end{array}$} & NHS Birmingham CrossCity & 721,400 & 1.84 & 1.52 & 1.38 & 1.62 & 1.48 & 1.41 & 133 & 1.54 & 1.42 & 1.67 & 141 & 35.2 \\
\hline & NHS Birmingham South and Central & 199,600 & 1.47 & 1.85 & 1.47 & 1.82 & 1.55 & 1.68 & 150 & 1.64 & 1.41 & 1.91 & 143 & 40.4 \\
\hline & NHS Dudley & 313,600 & 0.89 & 1.38 & 0.80 & 0.84 & 1.19 & 1.09 & 128 & 1.03 & 0.90 & 1.18 & 117 & 10.0 \\
\hline & NHS Sandwell and West Birmingham & 475,700 & 2.45 & 2.04 & 1.82 & 1.69 & 1.46 & 1.45 & 135 & 1.81 & 1.66 & 1.99 & 163 & 45.3 \\
\hline & NHS Solihull & 207,400 & 1.03 & 1.35 & 0.99 & 0.67 & 0.99 & 0.89 & 106 & 0.98 & 0.84 & 1.16 & 113 & 10.9 \\
\hline & NHS Walsall & 270,900 & 1.35 & 1.08 & 1.93 & 1.21 & 1.34 & 1.56 & 170 & 1.41 & 1.24 & 1.60 & 149 & 21.1 \\
\hline & NHS Wolverhampton & 251,000 & 1.42 & 1.12 & 1.46 & 1.15 & 1.49 & 1.05 & 112 & 1.28 & 1.12 & 1.47 & 132 & 32.0 \\
\hline
\end{tabular}


Table 1.3. Continued

\begin{tabular}{|c|c|c|c|c|c|c|c|c|c|c|c|c|c|c|}
\hline UK Area & CCG/HB & $\begin{array}{l}\text { Tot pop } \\
(2012)\end{array}$ & $\begin{array}{c}2008 \\
\mathrm{O} / \mathrm{E}\end{array}$ & $\begin{array}{c}2009 \\
\mathrm{O} / \mathrm{E}\end{array}$ & $\begin{array}{c}2010 \\
\mathrm{O} / \mathrm{E}\end{array}$ & $\begin{array}{l}2011 \\
\mathrm{O} / \mathrm{E}\end{array}$ & $\begin{array}{l}2012 \\
\mathrm{O} / \mathrm{E}\end{array}$ & $\mathrm{O} / \mathrm{E}$ & $\begin{array}{l}\text { Crude } \\
\text { rate } \\
\text { pmp }\end{array}$ & $\mathrm{O} / \mathrm{E}$ & $2008-$ & UCL & $\begin{array}{c}\text { Crude } \\
\text { rate } \\
\text { pmp }^{*}\end{array}$ & $\begin{array}{c}\% \\
\text { non- } \\
\text { White }\end{array}$ \\
\hline \multirow{10}{*}{$\begin{array}{l}\text { Derbyshire } \\
\text { and } \\
\text { Notting- } \\
\text { hamshire }\end{array}$} & NHS Erewash & 94,600 & 1.28 & 1.35 & 0.89 & 1.15 & 1.33 & 1.30 & 148 & 1.22 & 0.97 & 1.52 & 134 & 3.2 \\
\hline & NHS Hardwick & 108,900 & 1.04 & 1.02 & 0.40 & 0.70 & 0.85 & 0.76 & 92 & 0.80 & 0.62 & 1.02 & 93 & 1.8 \\
\hline & NHS Mansfield \& Ashfield & 192,500 & 0.91 & 1.09 & 0.92 & 0.75 & 0.83 & 0.82 & 93 & 0.88 & 0.74 & 1.06 & 98 & 2.5 \\
\hline & NHS Newark \& Sherwood & 115,900 & 0.97 & 0.95 & 0.97 & 1.30 & 0.93 & 0.49 & 60 & 0.93 & 0.75 & 1.17 & 111 & 2.4 \\
\hline & NHS North Derbyshire & 272,100 & 0.87 & 0.49 & 0.68 & 0.93 & 0.77 & 0.73 & 92 & 0.75 & 0.63 & 0.88 & 91 & 2.5 \\
\hline & NHS Nottingham City & 308,700 & 1.33 & 1.28 & 1.58 & 1.10 & 1.23 & 1.28 & 110 & 1.30 & 1.13 & 1.49 & 109 & 28.5 \\
\hline & NHS Nottingham North \& East & 146,200 & 0.80 & 1.21 & 0.87 & 0.78 & 0.72 & 0.70 & 82 & 0.85 & 0.68 & 1.05 & 96 & 6.2 \\
\hline & NHS Nottingham West & 110,700 & 1.04 & 1.10 & 0.97 & 0.55 & 1.08 & 1.22 & 145 & 0.99 & 0.79 & 1.24 & 114 & 7.3 \\
\hline & NHS Rushcliffe & 111,600 & 0.95 & 0.78 & 0.95 & 1.15 & 0.38 & 1.05 & 125 & 0.88 & 0.69 & 1.11 & 102 & 6.9 \\
\hline & NHS Southern Derbyshire & 515,300 & 1.44 & 1.07 & 0.96 & 1.04 & 1.13 & 0.88 & 97 & 1.09 & 0.98 & 1.20 & 116 & 11.0 \\
\hline \multirow[t]{8}{*}{ East Anglia } & NHS Cambridgeshire and Peterborough & 849,000 & 0.78 & 1.06 & 0.77 & 0.91 & 0.67 & 1.09 & 118 & 0.88 & 0.81 & 0.97 & 92 & 9.5 \\
\hline & NHS Great Yarmouth \& Waveney & 213,200 & 1.11 & 0.86 & 1.06 & 1.14 & 0.95 & 0.87 & 113 & 1.00 & 0.85 & 1.16 & 126 & 2.7 \\
\hline & NHS Ipswich and East Suffolk & 395,700 & 0.83 & 0.84 & 0.68 & 0.62 & 0.89 & 0.89 & 109 & 0.79 & 0.69 & 0.90 & 94 & 5.6 \\
\hline & NHS North Norfolk & 167,900 & 1.00 & 0.47 & 0.78 & 0.51 & 0.71 & 0.86 & 125 & 0.72 & 0.59 & 0.87 & 101 & 1.5 \\
\hline & NHS Norwich & 193,400 & 1.00 & 1.18 & 1.15 & 1.07 & 0.87 & 0.71 & 78 & 0.99 & 0.83 & 1.19 & 105 & 7.3 \\
\hline & NHS South Norfolk & 235,200 & 0.49 & 0.59 & 0.67 & 0.96 & 0.82 & 0.97 & 123 & 0.75 & 0.63 & 0.89 & 93 & 2.6 \\
\hline & NHS West Norfolk & 171,300 & 1.20 & 0.67 & 0.82 & 0.62 & 0.66 & 0.61 & 82 & 0.76 & 0.63 & 0.92 & 99 & 2.6 \\
\hline & NHS West Suffolk & 221,000 & 0.52 & 0.87 & 0.84 & 0.70 & 0.89 & 0.84 & 100 & 0.78 & 0.65 & 0.93 & 90 & 4.6 \\
\hline \multirow[t]{7}{*}{ Essex } & NHS Basildon and Brentwood & 250,500 & 0.95 & 0.89 & 0.83 & 1.03 & 1.24 & 0.86 & 96 & 0.97 & 0.83 & 1.13 & 104 & 7.1 \\
\hline & $\begin{array}{l}\text { NHS Castle Point, Rayleigh and } \\
\text { Rochford }\end{array}$ & 172,100 & 0.62 & 0.56 & 0.86 & 0.74 & 0.69 & 1.18 & 151 & 0.78 & 0.64 & 0.95 & 97 & 3.0 \\
\hline & NHS Mid Essex & 379,600 & 0.84 & 0.85 & 0.84 & 0.98 & 0.81 & 0.71 & 82 & 0.84 & 0.73 & 0.96 & 94 & 4.4 \\
\hline & NHS North East Essex & 314,300 & 1.64 & 0.86 & 0.98 & 1.25 & 0.95 & 0.86 & 105 & 1.09 & 0.96 & 1.23 & 129 & 5.5 \\
\hline & NHS Southend & 174,800 & 1.24 & 0.63 & 0.65 & 0.84 & 0.94 & 1.17 & 132 & 0.91 & 0.75 & 1.10 & 99 & 8.4 \\
\hline & NHS Thurrock & 159,500 & 1.50 & 0.47 & 1.17 & 1.20 & 0.79 & 0.91 & 88 & 1.00 & 0.82 & 1.23 & 94 & 14.1 \\
\hline & NHS West Essex & 290,000 & 0.42 & 0.83 & 0.65 & 0.72 & 1.19 & 0.98 & 110 & 0.80 & 0.68 & 0.94 & 87 & 8.2 \\
\hline \multirow{7}{*}{$\begin{array}{l}\text { Hertford- } \\
\text { shire and } \\
\text { the South } \\
\text { Midlands }\end{array}$} & NHS Bedfordshire & 419,200 & 0.71 & 0.86 & 0.90 & 0.74 & 1.00 & 1.06 & 117 & 0.88 & 0.77 & 1.00 & 93 & 11.2 \\
\hline & NHS Corby & 63,100 & 1.67 & 1.31 & 1.34 & 1.14 & 0.81 & 0.63 & 63 & 1.14 & 0.85 & 1.55 & 111 & 4.5 \\
\hline & NHS East and North Hertfordshire & 540,700 & 0.74 & 0.70 & 0.89 & 1.06 & 0.70 & 1.10 & 118 & 0.87 & 0.77 & 0.97 & 90 & 10.4 \\
\hline & NHS Herts Valleys & 569,900 & 1.06 & 0.92 & 0.86 & 0.78 & 0.89 & 0.93 & 98 & 0.91 & 0.81 & 1.01 & 93 & 14.6 \\
\hline & NHS Luton & 205,800 & 1.08 & 1.07 & 1.09 & 1.39 & 1.22 & 2.12 & 189 & 1.33 & 1.13 & 1.57 & 116 & 45.3 \\
\hline & NHS Milton Keynes & 257,900 & 0.91 & 0.90 & 1.05 & 0.98 & 1.14 & 0.91 & 85 & 0.98 & 0.83 & 1.16 & 89 & 19.6 \\
\hline & NHS Nene & 621,800 & 1.17 & 0.81 & 0.75 & 0.90 & 1.07 & 0.98 & 106 & 0.95 & 0.86 & 1.05 & 100 & 9.1 \\
\hline \multirow{7}{*}{$\begin{array}{l}\text { Leicester- } \\
\text { shire and } \\
\text { Lincolnshire }\end{array}$} & NHS East Leicestershire and Rutland & 319,500 & 0.60 & 0.54 & 0.71 & 0.69 & 0.98 & 0.93 & 113 & 0.75 & 0.64 & 0.87 & 87 & 9.8 \\
\hline & NHS Leicester City & 331,600 & 1.48 & 1.50 & 1.71 & 1.79 & 1.62 & 1.73 & 151 & 1.64 & 1.46 & 1.84 & 139 & 49.5 \\
\hline & NHS Lincolnshire East & 228,100 & 0.70 & 0.69 & 0.77 & 0.88 & 0.74 & 1.11 & 153 & 0.82 & 0.70 & 0.96 & 110 & 2.0 \\
\hline & NHS Lincolnshire West & 227,700 & 0.64 & 0.63 & 0.64 & 0.74 & 0.42 & 0.80 & 92 & 0.64 & 0.53 & 0.79 & 72 & 3.0 \\
\hline & NHS South Lincolnshire & 141,000 & 0.59 & 0.81 & 1.24 & 0.97 & 0.96 & 0.67 & 85 & 0.87 & 0.71 & 1.07 & 108 & 2.3 \\
\hline & NHS South West Lincolnshire & 122,000 & 0.70 & 0.96 & 0.91 & 0.95 & 0.68 & 0.86 & 107 & 0.85 & 0.67 & 1.06 & 101 & 2.3 \\
\hline & NHS West Leicestershire & 374,200 & 0.79 & 0.97 & 1.11 & 0.91 & 0.52 & 0.82 & 94 & 0.85 & 0.74 & 0.97 & 94 & 6.9 \\
\hline \multirow{8}{*}{$\begin{array}{l}\text { Shropshire } \\
\text { and } \\
\text { Staffordshire }\end{array}$} & NHS Cannock Chase & 132,800 & 1.04 & 0.48 & 1.12 & 1.15 & 0.81 & 0.99 & 113 & 0.93 & 0.75 & 1.16 & 103 & 2.4 \\
\hline & NHS East Staffordshire & 123,900 & 0.60 & 0.66 & 1.42 & 0.95 & 0.72 & 1.13 & 129 & 0.91 & 0.73 & 1.15 & 101 & 9.0 \\
\hline & NHS North Staffordshire & 213,200 & 0.89 & 1.11 & 0.69 & 1.10 & 0.58 & 0.84 & 103 & 0.87 & 0.73 & 1.03 & 103 & 3.5 \\
\hline & NHS Shropshire & 308,200 & 1.05 & 0.69 & 0.92 & 0.94 & 0.75 & 1.02 & 130 & 0.90 & 0.78 & 1.03 & 110 & 2.0 \\
\hline & $\begin{array}{l}\text { NHS South East Staffs and Seisdon } \\
\text { and Peninsular }\end{array}$ & 222,800 & 1.22 & 0.81 & 0.71 & 0.99 & 0.72 & 0.63 & 76 & 0.84 & 0.71 & 1.00 & 99 & 3.6 \\
\hline & NHS Stafford and Surrounds & 151,100 & 0.56 & 1.10 & 1.12 & 0.82 & 0.92 & 0.85 & 106 & 0.89 & 0.73 & 1.09 & 108 & 4.7 \\
\hline & NHS Stoke on Trent & 258,100 & 0.99 & 1.38 & 1.37 & 1.03 & 0.85 & 1.05 & 112 & 1.11 & 0.96 & 1.28 & 116 & 11.0 \\
\hline & NHS Telford \& Wrekin & 167,700 & 1.02 & 1.24 & 1.45 & 1.11 & 1.22 & 1.37 & 143 & 1.24 & 1.04 & 1.47 & 125 & 7.3 \\
\hline
\end{tabular}


Table 1.3. Continued

\begin{tabular}{|c|c|c|c|c|c|c|c|c|c|c|c|c|c|c|}
\hline UK Area & CCG/HB & $\begin{array}{l}\text { Tot pop } \\
\text { (2012) }\end{array}$ & $\begin{array}{c}2008 \\
\mathrm{O} / \mathrm{E}\end{array}$ & $\begin{array}{l}2009 \\
\text { O/E }\end{array}$ & $\begin{array}{c}2010 \\
\mathrm{O} / \mathrm{E}\end{array}$ & $\begin{array}{l}2011 \\
\mathrm{O} / \mathrm{E}\end{array}$ & $\begin{array}{l}2012 \\
\mathrm{O} / \mathrm{E}\end{array}$ & $\mathrm{O} / \mathrm{E}$ & $\begin{array}{l}3 \\
\text { Crude } \\
\text { rate } \\
\text { pmp }\end{array}$ & $\mathrm{O} / \mathrm{E}$ & 2008 & UCL & $\begin{array}{c}\text { Crude } \\
\text { rate } \\
\text { pmp }^{*}\end{array}$ & $\begin{array}{c}\% \\
\text { non- } \\
\text { White }\end{array}$ \\
\hline \multirow[t]{21}{*}{ London } & NHS Barking \& Dagenham & 190,600 & 1.78 & 1.42 & 1.38 & 1.67 & 2.07 & 1.71 & 136 & 1.67 & 1.42 & 1.96 & 130 & 41.7 \\
\hline & NHS Barnet & 364,000 & 1.43 & 1.25 & 1.78 & 1.45 & 1.53 & 1.30 & 124 & 1.46 & 1.30 & 1.63 & 134 & 35.9 \\
\hline & NHS Camden & 225,000 & 1.05 & 1.46 & 1.71 & 1.18 & 1.24 & 1.42 & 124 & 1.34 & 1.15 & 1.57 & 115 & 33.7 \\
\hline & NHS Haringey & 258,900 & 1.62 & 1.02 & 1.50 & 1.78 & 2.41 & 2.36 & 193 & 1.79 & 1.57 & 2.04 & 142 & 39.5 \\
\hline & NHS Havering & 239,700 & 0.78 & 0.69 & 0.35 & 1.18 & 1.06 & 0.81 & 92 & 0.81 & 0.68 & 0.97 & 89 & 12.3 \\
\hline & NHS Islington & 211,000 & 1.03 & 1.51 & 1.55 & 1.61 & 2.15 & 1.52 & 123 & 1.56 & 1.34 & 1.83 & 123 & 31.8 \\
\hline & NHS Newham & 314,100 & 1.68 & 2.15 & 2.40 & 2.30 & 2.08 & 2.40 & 169 & 2.17 & 1.93 & 2.44 & 149 & 71.0 \\
\hline & NHS Redbridge & 284,600 & 1.63 & 1.77 & 1.57 & 1.40 & 2.19 & 2.07 & 186 & 1.77 & 1.57 & 2.00 & 155 & 57.5 \\
\hline & NHS Central London (Westminster) & 161,000 & 1.22 & 1.40 & 1.37 & 1.39 & 1.32 & 1.49 & 143 & 1.37 & 1.14 & 1.63 & 127 & 36.2 \\
\hline & NHS Ealing & 340,700 & 1.44 & 2.34 & 2.02 & 1.92 & 2.28 & 1.68 & 150 & 1.95 & 1.75 & 2.16 & 169 & 51.0 \\
\hline & NHS Hammersmith and Fulham & 179,900 & 0.56 & 1.31 & 1.56 & 1.50 & 1.51 & 1.01 & 83 & 1.24 & 1.03 & 1.50 & 100 & 31.9 \\
\hline & NHS Harrow & 242,400 & 1.51 & 2.08 & 2.13 & 2.23 & 1.59 & 1.11 & 111 & 1.77 & 1.57 & 2.01 & 173 & 57.8 \\
\hline & NHS Hillingdon & 281,800 & 1.26 & 1.24 & 1.51 & 1.50 & 1.53 & 1.47 & 138 & 1.42 & 1.24 & 1.62 & 130 & 39.4 \\
\hline & NHS Hounslow & 259,100 & 1.10 & 1.63 & 1.86 & 1.88 & 1.79 & 2.10 & 185 & 1.73 & 1.52 & 1.97 & 148 & 48.6 \\
\hline & $\begin{array}{l}\text { NHS West London (Kensington } \\
\text { and Chelsea, Queen's Park and } \\
\text { Paddington) }\end{array}$ & 218,800 & 1.62 & 1.20 & 1.28 & 1.24 & 0.94 & 1.02 & 96 & 1.21 & 1.03 & 1.43 & 111 & 33.4 \\
\hline & NHS Bexley & 234,300 & 1.19 & 1.29 & 1.36 & 1.20 & 0.86 & 1.05 & 111 & 1.16 & 0.99 & 1.34 & 119 & 18.1 \\
\hline & NHS Bromley & 314,000 & 1.26 & 0.99 & 1.13 & 0.71 & 0.71 & 0.81 & 89 & 0.93 & 0.81 & 1.08 & 99 & 15.7 \\
\hline & NHS Richmond & 189,100 & 0.66 & 0.81 & 0.89 & 0.70 & 0.80 & 0.95 & 95 & 0.80 & 0.65 & 0.99 & 78 & 14.0 \\
\hline & NHS Southwark & 293,500 & 2.14 & 1.54 & 1.95 & 2.06 & 1.84 & 2.28 & 177 & 1.97 & 1.74 & 2.22 & 149 & 45.8 \\
\hline & NHS Sutton & 193,600 & 1.44 & 0.99 & 1.44 & 1.30 & 1.55 & 0.86 & 88 & 1.26 & 1.07 & 1.48 & 125 & 21.4 \\
\hline & NHS Wandsworth & 308,300 & 1.42 & 1.99 & 1.50 & 1.23 & 1.27 & 0.93 & 75 & 1.39 & 1.21 & 1.59 & 109 & 28.6 \\
\hline Bath, & NHS Bath and North East Somerset & 177,600 & 0.74 & 1.24 & 0.63 & 0.56 & 0.91 & 0.95 & 107 & 0.84 & 0.69 & 1.02 & 92 & 5.4 \\
\hline Gloucester- & NHS Gloucestershire & 602,200 & 0.63 & 1.14 & 0.90 & 0.89 & 1.18 & 0.73 & 86 & 0.91 & 0.82 & 1.01 & 105 & 4.6 \\
\hline shire, and & NHS Swindon & 217,200 & 1.08 & 1.07 & 1.04 & 1.15 & 1.23 & 0.94 & 97 & 1.09 & 0.92 & 1.28 & 108 & 10.0 \\
\hline Wiltshire & NHS Wiltshire & 476,800 & 0.83 & 0.78 & 0.81 & 0.64 & 0.49 & 0.79 & 92 & 0.72 & 0.64 & 0.82 & 82 & 3.4 \\
\hline Bristol, North & NHS Bristol & 432,500 & 1.53 & 1.28 & 1.49 & 1.41 & 1.24 & 1.36 & 127 & 1.38 & 1.24 & 1.54 & 126 & 16.0 \\
\hline Somerset, & NHS North Somerset & 204,400 & 1.28 & 0.96 & 0.99 & 0.88 & 0.99 & 1.05 & 132 & 1.02 & 0.87 & 1.20 & 125 & 2.7 \\
\hline Somerset and & NHS Somerset & 535,000 & 0.79 & 1.08 & 1.09 & 0.83 & 0.67 & 0.56 & 71 & 0.84 & 0.75 & 0.93 & 103 & 2.0 \\
\hline $\begin{array}{l}\text { South Glouces- } \\
\text { tershire }\end{array}$ & NHS South Gloucestershire & 266,100 & 0.92 & 0.66 & 1.09 & 0.62 & 0.82 & 1.17 & 132 & 0.88 & 0.75 & 1.03 & 96 & 5.0 \\
\hline Devon, & NHS Kernow & 540,200 & 0.91 & 1.07 & 0.89 & 0.81 & 0.96 & 0.87 & 113 & 0.92 & 0.83 & 1.02 & 115 & 1.8 \\
\hline Cornwall and & NHS North, East, West Devon & 869,400 & 1.10 & 1.06 & 1.00 & 0.92 & 1.00 & 0.84 & 104 & 0.98 & 0.91 & 1.07 & 117 & 3.0 \\
\hline Isles of Scilly & NHS South Devon and Torbay & 273,300 & 1.43 & 0.87 & 1.26 & 0.89 & 1.07 & 1.00 & 135 & 1.08 & 0.95 & 1.23 & 142 & 2.1 \\
\hline
\end{tabular}


Table 1.3. Continued

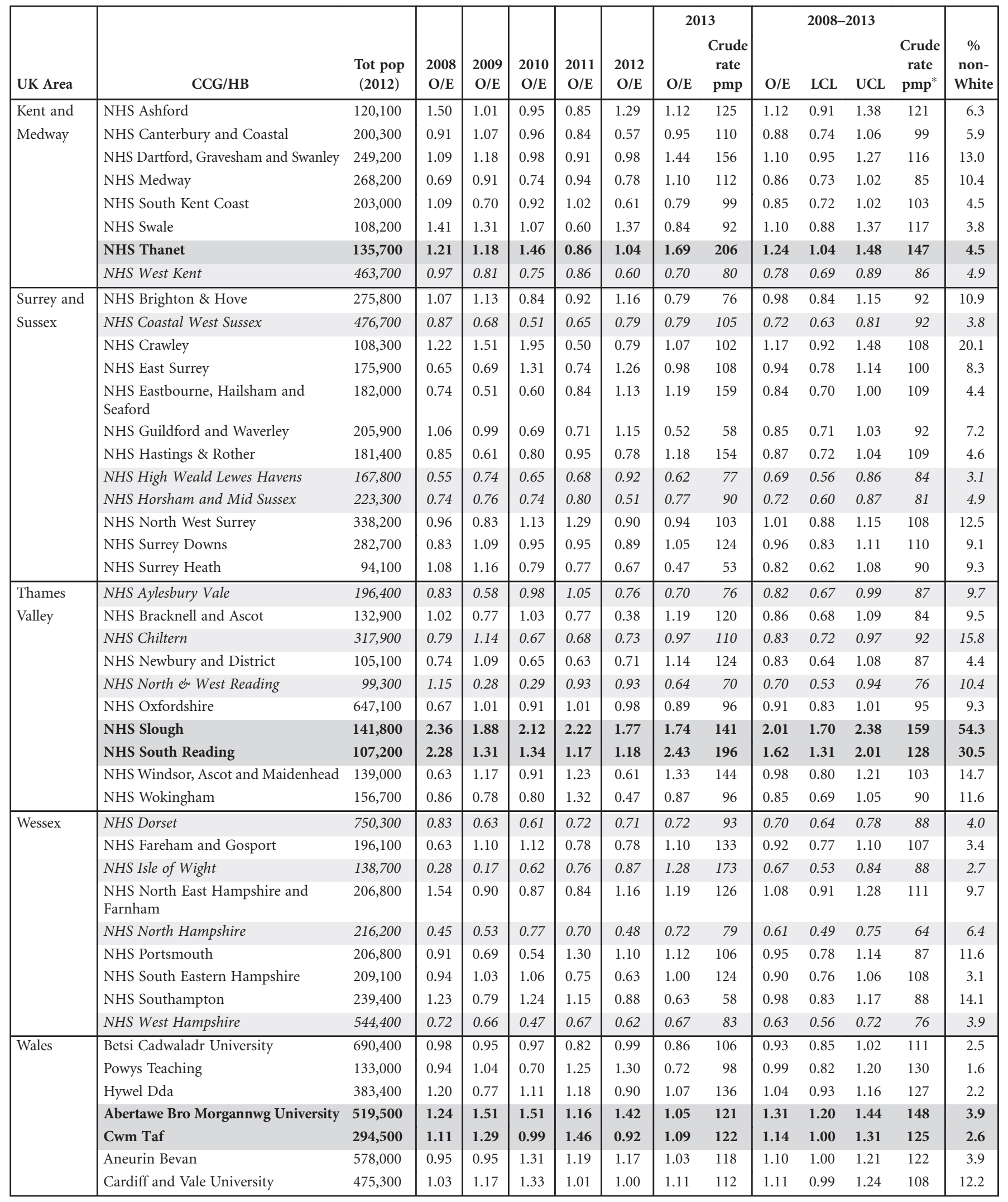


Table 1.3. Continued

\begin{tabular}{|c|c|c|c|c|c|c|c|c|c|c|c|c|c|c|}
\hline UK Area & CCG/HB & $\begin{array}{c}\text { Tot pop } \\
(2012)\end{array}$ & $\begin{array}{c}2008 \\
\mathrm{O} / \mathrm{E}\end{array}$ & $\begin{array}{c}2009 \\
\mathrm{O} / \mathrm{E}\end{array}$ & $\begin{array}{l}2010 \\
\mathrm{O} / \mathrm{E}\end{array}$ & $\begin{array}{c}2011 \\
\mathrm{O} / \mathrm{E}\end{array}$ & $\begin{array}{c}2012 \\
\mathrm{O} / \mathrm{E}\end{array}$ & $\mathrm{O} / \mathrm{E}$ & $\begin{array}{l}13 \\
\text { Crude } \\
\text { rate } \\
\text { pmp }\end{array}$ & $\mathrm{O} / \mathrm{E}$ & 2008 & UCL & $\begin{array}{c}\text { Crude } \\
\text { rate } \\
\text { pmp }^{*}\end{array}$ & $\begin{array}{c}\% \\
\text { non- } \\
\text { White }\end{array}$ \\
\hline \multirow[t]{8}{*}{ Scotland } & Ayrshire and Arran & 373,200 & 0.88 & 0.88 & 1.11 & 0.81 & 0.94 & 0.99 & 121 & 0.93 & 0.82 & 1.06 & 111 & 1.2 \\
\hline & Borders & 113,700 & 1.07 & 0.97 & 1.06 & 0.55 & 0.48 & 0.47 & 62 & 0.76 & 0.60 & 0.97 & 97 & 1.3 \\
\hline & Dumfries and Galloway & 150,800 & 1.11 & 1.08 & 0.58 & 0.56 & 1.01 & 0.45 & 60 & 0.80 & 0.65 & 0.98 & 103 & 1.2 \\
\hline & Grampian & 573,400 & 0.91 & 0.85 & 0.85 & 0.82 & 0.82 & 0.91 & 101 & 0.86 & 0.77 & 0.96 & 92 & 4.0 \\
\hline & Greater Glasgow and Clyde & $1,217,000$ & 0.95 & 0.99 & 0.87 & 1.07 & 1.09 & 0.95 & 102 & 0.99 & 0.92 & 1.06 & 103 & 7.3 \\
\hline & Highland & 319,800 & 0.76 & 0.75 & 0.63 & 0.51 & 0.63 & 0.62 & 78 & 0.65 & 0.56 & 0.76 & 79 & 1.3 \\
\hline & Lanarkshire & 572,500 & 0.74 & 0.86 & 1.01 & 0.82 & 1.12 & 0.85 & 94 & 0.90 & 0.81 & 1.00 & 97 & 2.0 \\
\hline & Lothian & 843,700 & 0.97 & 0.85 & 0.62 & 0.73 & 0.74 & 0.60 & 63 & 0.75 & 0.68 & 0.83 & 76 & 5.6 \\
\hline \multirow{5}{*}{$\begin{array}{l}\text { Northern } \\
\text { Ireland }\end{array}$} & Belfast & 348,300 & 1.02 & 0.77 & 1.30 & 1.05 & 1.67 & 1.15 & 115 & 1.16 & 1.02 & 1.32 & 113 & 3.2 \\
\hline & Northern & 465,500 & 1.16 & 0.81 & 1.15 & 1.26 & 1.15 & 1.00 & 105 & 1.09 & 0.97 & 1.22 & 111 & 1.2 \\
\hline & Southern & 363,100 & 0.99 & 0.77 & 1.03 & 1.29 & 0.82 & 0.83 & 80 & 0.96 & 0.83 & 1.10 & 89 & 1.2 \\
\hline & South Eastern & 350,100 & 0.87 & 0.66 & 0.70 & 0.93 & 0.79 & 0.90 & 97 & 0.81 & 0.70 & 0.94 & 84 & 1.3 \\
\hline & Western & 296,600 & 0.83 & 1.21 & 0.84 & 1.09 & 0.56 & 0.99 & 98 & 0.92 & 0.79 & 1.08 & 88 & 1.0 \\
\hline
\end{tabular}

between areas. From the analysis using all six years combined, 49 areas were significantly high and 66 were significantly low out of a total of 237 areas. The standardised incidence ratios ranged from 0.45 to 2.25 (IQR 0.82, 1.10). As previously reported, urban areas with high percentages of non-White residents tended to have high incidence rates. Figure 1.2 shows the strong positive correlation between the standardised incidence

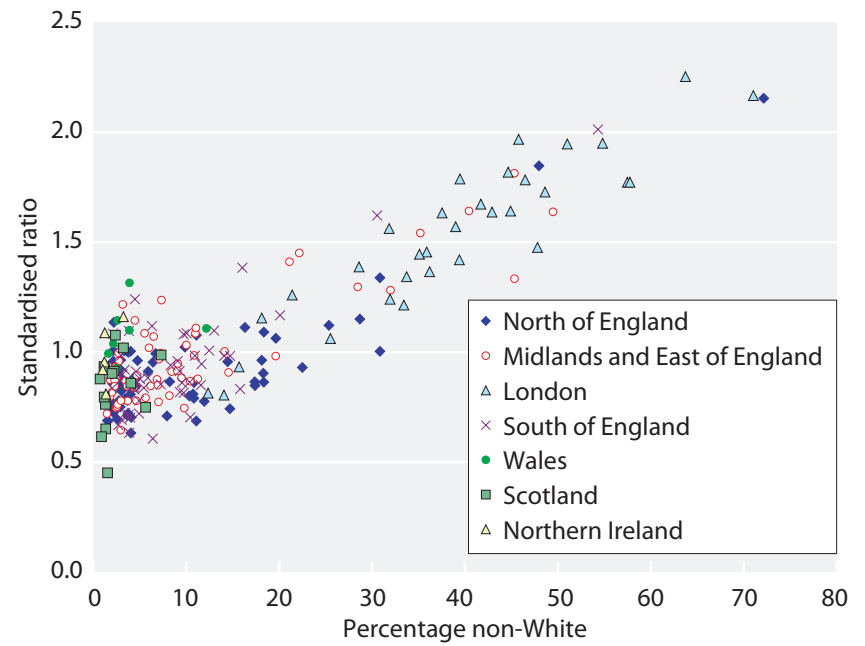

Fig. 1.2. Age/gender standardised incidence ratio (2008-2013) by percentage non-White ratio and the percentage of the $\mathrm{CCG} / \mathrm{HB}$ population that was non-White.

\section{Centre level}

The number of new patients starting RRT at each renal centre from 2008 to 2013 is shown in table 1.4. The table also shows centre level incidence rates (per million population) for 2013. For most centres there was a lot of variability in the numbers of incident patients from one year to the next making it hard to see any underlying trend. Some centres have had an increase in new patients over time and others have fallen. The variation may reflect chance fluctuation, the introduction of new centres, changes in catchment populations or in completeness of reporting. Variation over time may also be due to changing incidence of established renal failure (increases in underlying disease prevalence, survival from comorbid conditions and recognition of ERF), changes to treatment thresholds such as a greater emphasis on pre-emptive transplantation or the introduction of conservative care programmes. Analysis of CKD stage 5 patients not yet on RRT is required to explore some of these underlying mechanisms for centre level incidence rate changes.

There was a fall of approximately $5 \%$ in the number of new patients for Scotland between 2008 and 2013. There was an increase of $6 \%$ in new patients for England 
Table 1.4. Number of patients starting RRT by renal centre 2008-2013

\begin{tabular}{|c|c|c|c|c|c|c|c|c|c|}
\hline \multirow[b]{2}{*}{ Centre } & \multicolumn{6}{|c|}{ Year } & \multirow{2}{*}{$\begin{array}{l}\text { Catchment } \\
\text { population } \\
\text { (millions) }\end{array}$} & \multirow{2}{*}{$\begin{array}{c}2013 \\
\text { crude rate } \\
\text { pmp }^{\mathrm{a}}\end{array}$} & \multirow[b]{2}{*}{$(95 \% \mathrm{CI})$} \\
\hline & 2008 & 2009 & 2010 & 2011 & 2012 & 2013 & & & \\
\hline \multicolumn{10}{|l|}{ England } \\
\hline B Heart & 105 & 99 & 94 & 113 & 102 & 99 & 0.74 & 134 & $(108-161)$ \\
\hline B QEH & 267 & 256 & 198 & 216 & 213 & 191 & 1.70 & 112 & $(96-128)$ \\
\hline Basldn & 41 & 28 & 34 & 44 & 53 & 32 & 0.42 & 77 & $(50-104)$ \\
\hline Bradfd & 62 & 57 & 67 & 60 & 69 & 62 & 0.65 & 95 & $(71-119)$ \\
\hline Brightn & 118 & 117 & 106 & 119 & 135 & 139 & 1.30 & 107 & $(89-125)$ \\
\hline Bristol & 175 & 157 & 169 & 140 & 148 & 173 & 1.44 & 120 & $(102-138)$ \\
\hline Camb & 94 & 134 & 106 & 122 & 125 & 139 & 1.16 & 120 & $(100-140)$ \\
\hline Carlis & 30 & 28 & 22 & 28 & 19 & 41 & 0.32 & 128 & $(89-167)$ \\
\hline Carsh & 210 & 202 & 216 & 207 & 243 & 231 & 1.91 & 121 & $(105-136)$ \\
\hline Chelms & 36 & 51 & 45 & 47 & 46 & 42 & 0.51 & 82 & $(57-107)$ \\
\hline Colchr & 58 & 21 & 32 & 44 & 29 & 30 & 0.30 & 100 & $(64-136)$ \\
\hline Covnt $^{\mathrm{b}}$ & 113 & 115 & 114 & 110 & 113 & 96 & 0.89 & 108 & $(86-129)$ \\
\hline Derby & 96 & 77 & 78 & 76 & 79 & 74 & 0.70 & 105 & $(81-129)$ \\
\hline Donc & 26 & 40 & 45 & 43 & 40 & 60 & 0.41 & 146 & $(109-183)$ \\
\hline Dorset & 82 & 73 & 72 & 79 & 73 & 74 & 0.86 & 86 & $(66-105)$ \\
\hline Dudley & 47 & 67 & 43 & 43 & 56 & 47 & 0.44 & 106 & $(76-137)$ \\
\hline Exeter $^{b}$ & 135 & 145 & 139 & 112 & 135 & 108 & 1.09 & 99 & $(80-118)$ \\
\hline Glouc & 46 & 79 & 61 & 58 & 76 & 54 & 0.59 & 92 & $(67-116)$ \\
\hline Hull & 110 & 99 & 86 & 109 & 97 & 92 & 1.02 & 90 & (72-109) \\
\hline Ipswi & 38 & 38 & 33 & 29 & 43 & 39 & 0.40 & 98 & $(67-128)$ \\
\hline Kent & 138 & 126 & 132 & 121 & 115 & 145 & 1.22 & 118 & $(99-138)$ \\
\hline L Barts & 206 & 236 & 201 & 251 & 268 & 291 & 1.83 & 159 & $(141-177)$ \\
\hline L Guys & 161 & 172 & 144 & 123 & 129 & 130 & 1.08 & 120 & $(99-141)$ \\
\hline L Kings & 151 & 127 & 144 & 139 & 124 & 162 & 1.17 & 138 & $(117-160)$ \\
\hline L Rfree & 172 & 170 & 203 & 220 & 237 & 228 & 1.52 & 150 & $(131-170)$ \\
\hline L St.G ${ }^{b}$ & 99 & 110 & 85 & 72 & 90 & 81 & 0.80 & 102 & $(79-124)$ \\
\hline L West & 294 & 357 & 365 & 365 & 355 & 303 & 2.40 & 126 & $(112-141)$ \\
\hline Leeds & 160 & 149 & 125 & 158 & 154 & 184 & 1.67 & 110 & $(94-126)$ \\
\hline Leic & 242 & 227 & 244 & 266 & 236 & 291 & 2.44 & 119 & $(106-133)$ \\
\hline Liv Ain & 42 & 38 & 50 & 58 & 63 & 66 & 0.48 & 136 & $(103-169)$ \\
\hline Liv Roy & 102 & 110 & 99 & 112 & 104 & 94 & 1.00 & 94 & $(75-113)$ \\
\hline M RI & 130 & 146 & 161 & 155 & 161 & 200 & 1.53 & 131 & $(113-149)$ \\
\hline Middlbr & 95 & 96 & 100 & 101 & 120 & 108 & 1.00 & 108 & $(87-128)$ \\
\hline Newc & 99 & 97 & 91 & 97 & 104 & 95 & 1.12 & 85 & $(68-102)$ \\
\hline Norwch & 84 & 71 & 85 & 85 & 74 & 76 & 0.79 & 97 & (75-118) \\
\hline Nottm & 115 & 133 & 116 & 114 & 101 & 113 & 1.09 & 104 & $(85-123)$ \\
\hline Oxford & 147 & 174 & 165 & 177 & 170 & 166 & 1.69 & 98 & $(83-113)$ \\
\hline Plymth $^{c}$ & 69 & 57 & 56 & 60 & 55 & 63 & 0.47 & 134 & $(101-167)$ \\
\hline Ports & 170 & 149 & 149 & 187 & 160 & 198 & 2.02 & 98 & $(84-111)$ \\
\hline Prestn & 113 & 146 & 123 & 140 & 146 & 151 & 1.49 & 101 & $(85-117)$ \\
\hline Redng & 103 & 94 & 89 & 103 & 73 & 117 & 0.91 & 129 & $(105-152)$ \\
\hline Salford ${ }^{b}$ & 138 & 125 & 149 & 132 & 134 & 122 & 1.49 & 82 & $(67-96)$ \\
\hline Sheff & 179 & 149 & 142 & 135 & 157 & 137 & 1.37 & 100 & $(83-117)$ \\
\hline Shrew & 59 & 48 & 58 & 61 & 58 & 61 & 0.50 & 122 & $(91-152)$ \\
\hline Stevng ${ }^{b}$ & 103 & 98 & 107 & 110 & 109 & 130 & 1.20 & 108 & $(89-127)$ \\
\hline Sthend & 36 & 23 & 27 & 29 & 26 & 42 & 0.32 & 133 & $(92-173)$ \\
\hline Stoke & 79 & 108 & 95 & 91 & 74 & 100 & 0.89 & 112 & $(90-134)$ \\
\hline Sund & 45 & 64 & 54 & 57 & 71 & 49 & 0.62 & 79 & $(57-101)$ \\
\hline Truro & 41 & 58 & 46 & 38 & 49 & 46 & 0.41 & 111 & (79-144) \\
\hline Wirral & 39 & 63 & 60 & 60 & 44 & 68 & 0.57 & 119 & $(91-147)$ \\
\hline Wolve & 89 & 65 & 106 & 77 & 87 & 88 & 0.67 & 132 & $(104-159)$ \\
\hline York & 37 & 43 & 38 & 51 & 53 & 36 & 0.49 & 73 & $(49-97)$ \\
\hline
\end{tabular}


Table 1.4. Continued

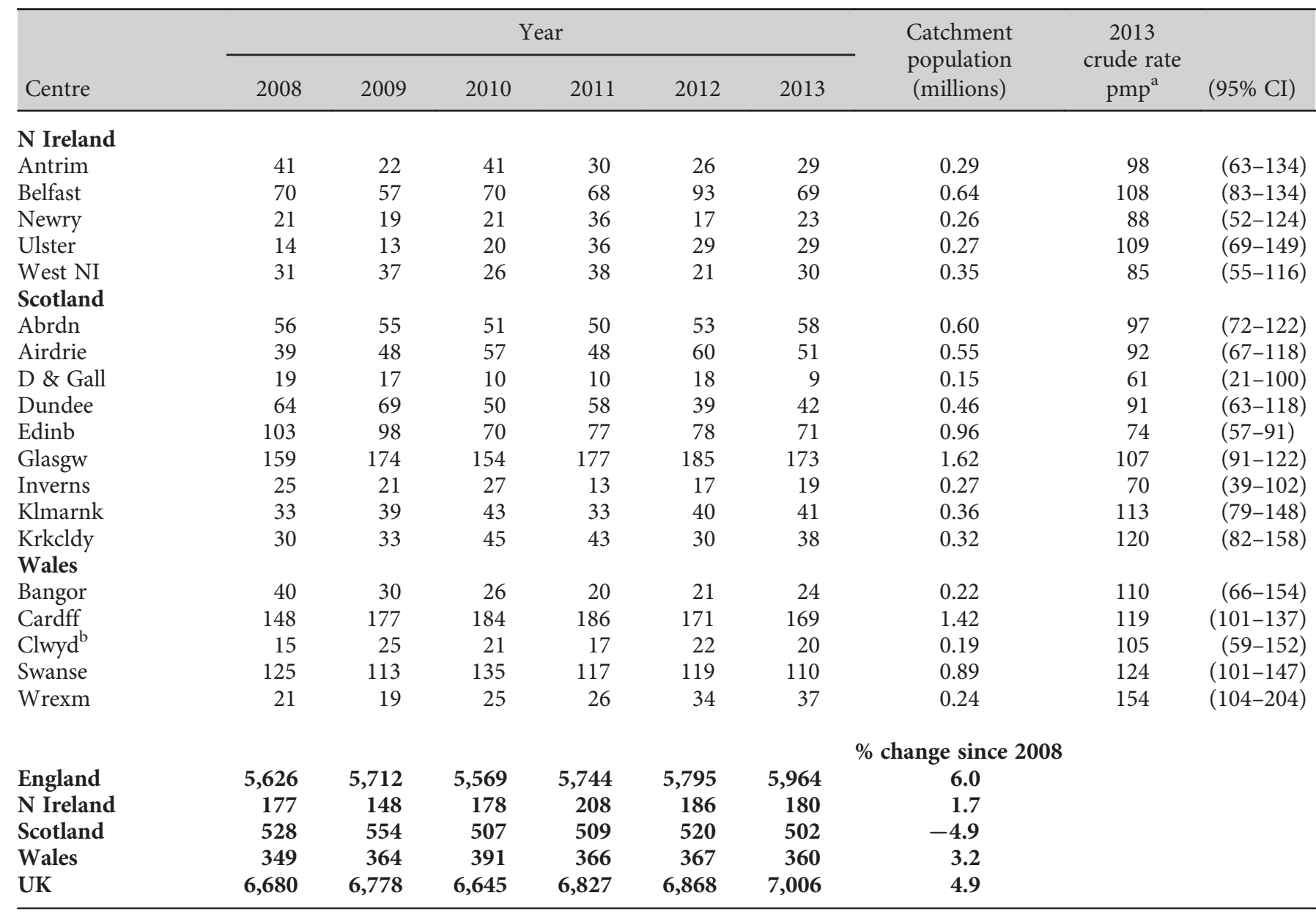

${ }^{\mathrm{a}} \mathrm{pmp}$ - per million population

${ }^{\mathrm{b}}$ Subsequent to closing the 2013 database several centres reported a variation to the numbers returned for 2013 . Tables 1.2 and 1.4 (but not the remainder of this chapter) reflect these revisions (Covnt $(+9)$, Exeter $(+6)$, L St.G $(+5)$, Salford $(+11)$, Stevng $(-29)$, Clwyd $(+6)$ )

'In last year's report the data included 47 incident patients for Plymouth for 2012 but the centre advised the UKRR that the number was 75 and an adjustment was made to the summary tables. After extensive data validation work the data now shows that there were 55 incident patients for 2012

between 2008 and 2013. Across all four countries the change between 2008 and 2013 was an increase of 4.9\%.

\section{Demographics and clinical characteristics of patients starting RRT}

\section{Methods}

Age, gender, primary renal disease, ethnic origin and treatment modality were examined for patients starting RRT. Individual EDTA codes for primary diagnoses were grouped into eight categories, the details are given in appendix $\mathrm{H}$ : Ethnicity and ERA-EDTA Coding (www.renalreg.org).

Most centres electronically upload ethnicity coding to their renal information technology (IT) system from the hospital
Patient Administration System (PAS). Ethnicity coding in these PAS systems is based on self-reported ethnicity. For the remaining centres, ethnicity coding is performed by clinical staff and recorded directly into the renal IT system (using a variety of coding systems). For all these analyses, data on ethnic origin were grouped into White, South Asian, Black, Chinese or Other. The details of regrouping of the PAS codes into the above ethnic categories are provided in appendix $\mathrm{H}$ : Ethnicity and ERAEDTA Coding (www.renalreg.org). Chi-squared, Fisher's exact, ANOVA and Kruskal Wallis tests were used as appropriate to test for significant differences.

Estimated glomerular filtration rate (eGFR) at the start of RRT was studied amongst patients with eGFR data within 14 days before the start of RRT. The eGFR was calculated using the abbreviated 4 variable MDRD study equation [2]. For the purpose of the eGFR calculation, patients who had missing ethnicity but a valid serum creatinine measurement were classed as White. The eGFR values were log transformed in order to normalise the data. 


\section{Results}

Age

Overall, incidence rates have plateaued in the last eight years (figure 1.3). Figure 1.4 shows RRT incidence rates for 2013 by age group and gender. For women, the peak rate was in the 75-79 age group and in men in the $80-84$ age group. Showing numbers starting RRT (rather than rates), figure 1.5 shows that the 65-74 age group contained the most incident patients for both HD and PD.

In 2013, the median age of patients starting renal replacement therapy was 64.5 years (table 1.5) and this has changed little over the last six years (data not shown). The median age at start was 67.1 years for patients starting on HD, 59.7 for patients starting on $\mathrm{PD}$ and 49.7 for those having a pre-emptive transplant

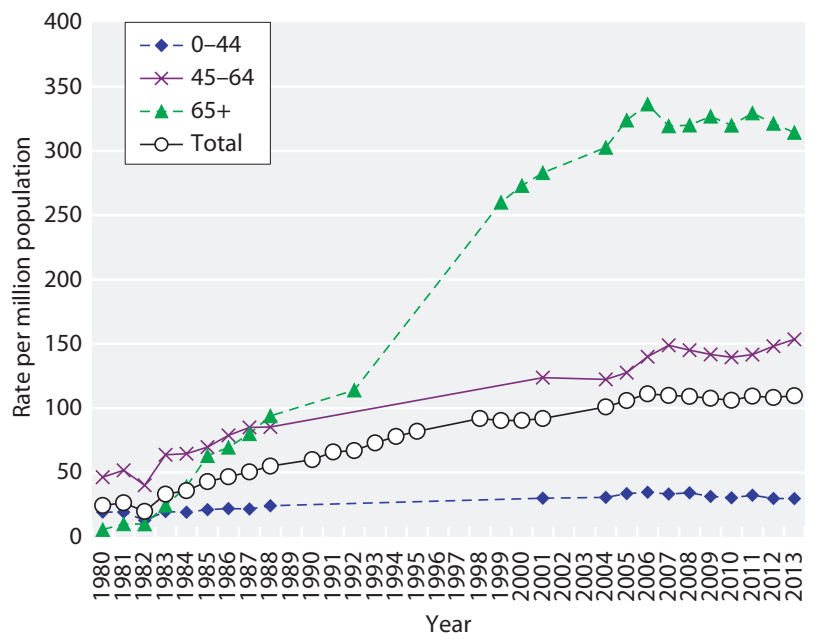

Fig. 1.3. RRT incidence rates between 1980 and 2013

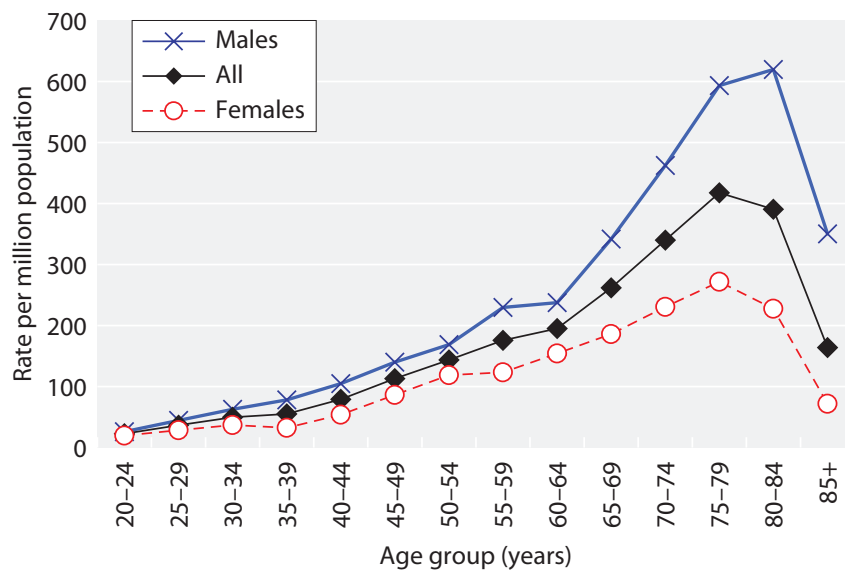

Fig. 1.4. RRT incidence rates in 2013 by age and gender

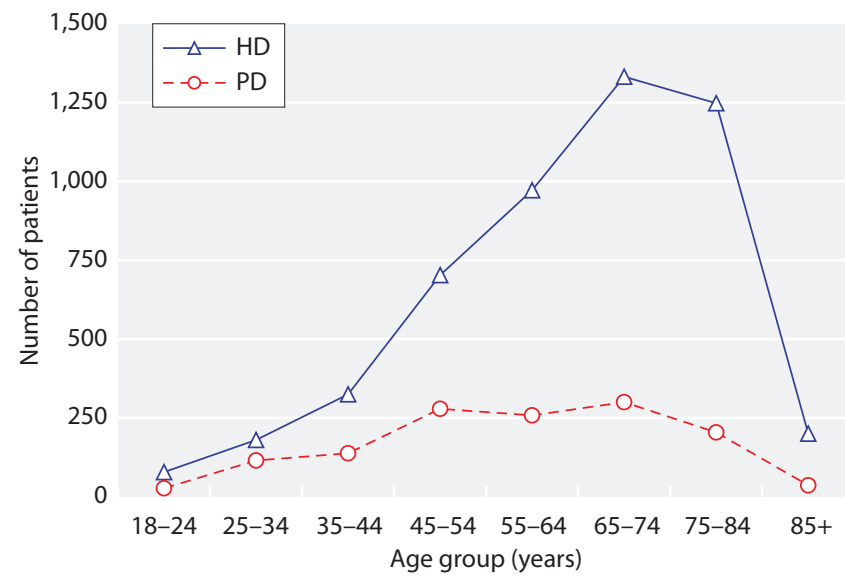

Fig. 1.5. Number of incident dialysis patients in 2013, by age group and initial dialysis modality

Table 1.5. Median, inter-quartile range and $90 \%$ range of the age of patients starting renal replacement therapy in 2013 by country

\begin{tabular}{lccc}
\hline Country & Median & IQR & 90\% range \\
\hline England & 64.2 & $(51.0-74.6)$ & $(31.3-83.9)$ \\
N Ireland & 66.7 & $(50.9-75.0)$ & $(32.0-82.8)$ \\
Scotland & 64.1 & $(51.1-74.1)$ & $(31.7-82.8)$ \\
Wales & 68.9 & $(57.2-75.9)$ & $(34.4-84.6)$ \\
UK & $\mathbf{6 4 . 5}$ & $\mathbf{( 5 1 . 2 - 7 4 . 7 )}$ & $\mathbf{( 3 1 . 6 - 8 3 . 9 )}$ \\
\hline
\end{tabular}

(table 1.6). The median age of non-White patients (57.0 years) was considerably lower than for White patients (66.0 years) reflecting CKD differences and the younger age distribution of ethnic minority populations in general compared with the White population (in the 2011 census data for England and Wales 5.3\% of ethnic minorities were over 65 years old compared to $18.3 \%$ of Whites) [3]. The median age of new patients with diabetes was similar to the overall median and has not varied greatly over the last five years.

There were large differences between centres in the median age of incident patients (figure 1.6) reflecting differences in the age and ethnic structure of the catchment populations and also, particularly in smaller

Table 1.6. Median, inter-quartile range and $90 \%$ range of the age of patients starting renal replacement therapy in 2013 by initial treatment modality

\begin{tabular}{lccc}
\hline Treatment & Median & IQR & $90 \%$ range \\
\hline HD & 67.1 & $(54.6-76.2)$ & $(34.7-84.5)$ \\
PD & 59.7 & $(47.2-71.5)$ & $(29.3-82.5)$ \\
Transplant & 49.7 & $(40.6-59.3)$ & $(25.1-69.2)$ \\
\hline
\end{tabular}




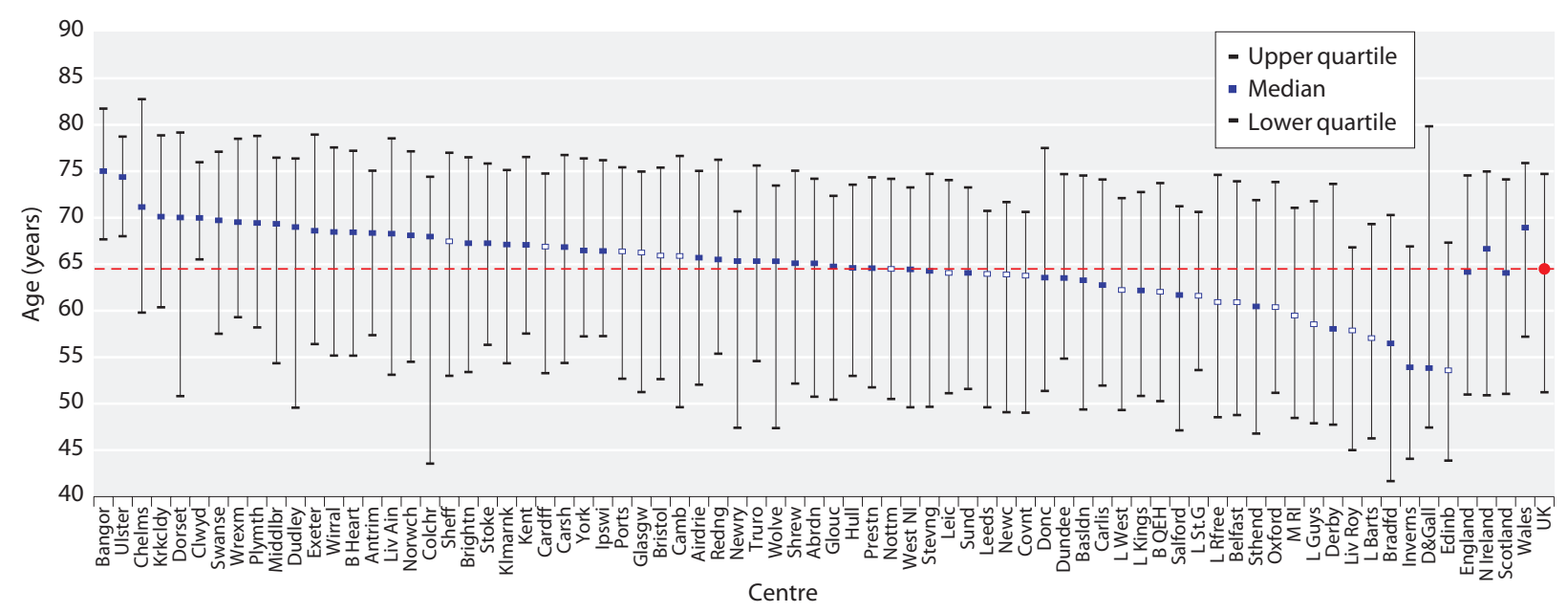

Fig. 1.6. Median age of incident RRT patients by centre in 2013 White points indicate transplant centres

centres, chance fluctuations. The median age of patients starting treatment at transplant centres was 62.8 years (IQR 49.8, 73.8) and at non-transplanting centres 65.7 years (IQR 52.4, 75.4) $(p<0.0001)$.

Averaged over 2008-2013, crude CCG/HB incidence rates in the over 75 years age group varied from 99 per million age related population (pmarp) in Shetland to 947 pmarp in NHS Brent (data not shown). Excluding two areas which had much higher rates than the rest, there was 7.3 -fold variation (99 pmarp to 722 pmarp). The wide range of treatment rates suggests that there was geographical variation in the prevalence of comorbid and predisposing renal conditions as well as uncertainty within the renal community about the suitability of older patients for dialysis. The 7.3-fold variation between CCG/HBs seen in the over $75 \mathrm{~s}$ was much greater than the 2.7 -fold variation (64 pmp to $173 \mathrm{pmp}$ ) after excluding two outliers seen in the overall analysis although some of this difference is likely to be due to the smaller numbers included in the over 75 analysis.

\section{Gender}

There continued to be more men than women starting RRT in every age group (figure 1.7). The overall breakdown was $63.4 \%$ male, $36.6 \%$ female equating to a $\mathrm{M}: \mathrm{F}$ ratio of 1.73 .

\section{Ethnicity}

As in previous reports, Scotland is not included in this section as ethnicity completeness was low. Across centres in England, Wales and Northern Ireland the average completeness fell slightly in 2013 to $95.2 \%$ (vs. $98.1 \%$ for 2012). This was in large part due to one centre
(Carshalton) which fell from a completeness of 85.9 to $54.1 \%$. Completeness was $80 \%$ or more for all the other centres for 2013 (table 1.7) and was over 90\% for all but seven centres. Ten centres reported no non-White patients starting in 2013 whilst some London centres reported over $50 \%$.

\section{Primary renal diagnosis}

The breakdown of primary renal diagnosis (PRD) by centre is shown in table 1.8. The information was missing for $9.5 \%$ of patients. Fifty-eight centres provided data on over $90 \%$ of incident patients and 36 of these centres had $100 \%$ completeness. There was only a small amount of missing data for Wales, Northern Ireland and Scotland, whilst England had 11.0\% missing (up from 7.4\% for 2012). The overall percentage missing was up on 2012

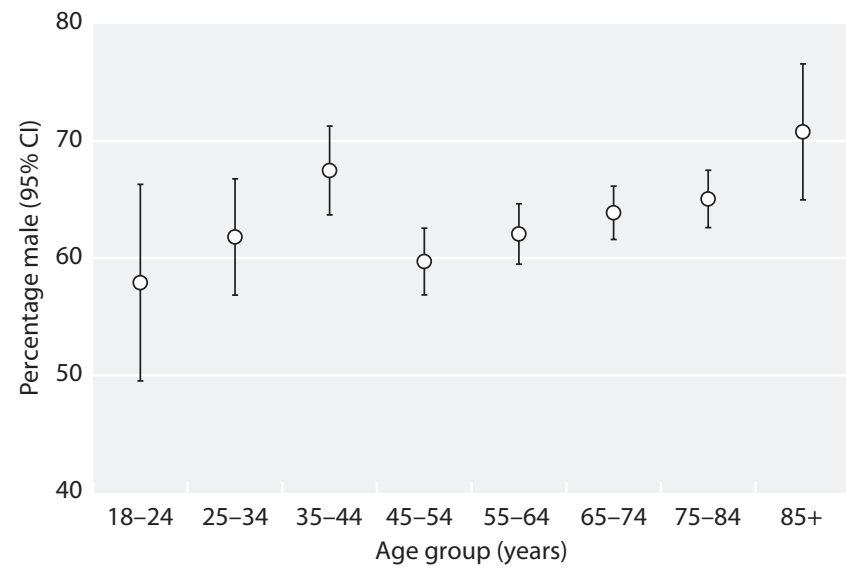

Fig. 1.7. Percentage of patients starting RRT in 2013 who were male, by age group 
Table 1.7. Percentage of incident RRT patients (2013) in different ethnic groups by centre

\begin{tabular}{|c|c|c|c|c|c|c|c|}
\hline \multirow[b]{2}{*}{ Centre } & \multirow{2}{*}{$\begin{array}{c}\% \text { data not } \\
\text { available }\end{array}$} & \multirow{2}{*}{$\begin{array}{c}N \text { with } \\
\text { data }\end{array}$} & \multicolumn{5}{|c|}{ Percentage in each ethnic group } \\
\hline & & & White & South Asian & Black & Chinese & Other \\
\hline \multicolumn{8}{|l|}{ England } \\
\hline B Heart & 0.0 & 99 & 55.6 & 34.3 & 7.1 & 2.0 & 1.0 \\
\hline B QEH & 0.0 & 191 & 64.9 & 22.0 & 10.5 & & 2.6 \\
\hline Basldn & 0.0 & 32 & 90.6 & 6.3 & & 3.1 & \\
\hline Bradfd & 0.0 & 62 & 53.2 & 43.5 & 1.6 & & 1.6 \\
\hline Brightn & 1.4 & 137 & 90.5 & 2.9 & 1.5 & & 5.1 \\
\hline Bristol & 2.3 & 169 & 88.8 & 4.1 & 2.4 & 0.6 & 4.1 \\
\hline Camb & 6.5 & 130 & 96.2 & 1.5 & 1.5 & & 0.8 \\
\hline Carlis & 2.4 & 40 & 95.0 & & 2.5 & & 2.5 \\
\hline Carsh & 45.9 & 125 & 72.0 & 17.6 & 4.8 & 0.8 & 4.8 \\
\hline Chelms & 19.0 & 34 & 88.2 & 5.9 & & 5.9 & \\
\hline Colchr & 3.3 & 29 & 100.0 & & & & \\
\hline Covnt & 0.0 & 87 & 83.9 & 12.6 & 3.4 & & \\
\hline Derby & 8.1 & 68 & 89.7 & 5.9 & 2.9 & & 1.5 \\
\hline Donc & 0.0 & 60 & 91.7 & 3.3 & 1.7 & & 3.3 \\
\hline Dorset & 0.0 & 74 & 95.9 & 1.4 & 1.4 & & 1.4 \\
\hline Dudley & 4.3 & 45 & 88.9 & 8.9 & 2.2 & & \\
\hline Exeter & 1.0 & 101 & 100.0 & & & & \\
\hline Glouc & 0.0 & 54 & 92.6 & 3.7 & 1.9 & & 1.9 \\
\hline Hull & 3.3 & 89 & 97.8 & 2.2 & & & \\
\hline Ipswi & 15.4 & 33 & 100.0 & & & & \\
\hline Kent & 2.1 & 142 & 96.5 & 0.7 & 1.4 & 0.7 & 0.7 \\
\hline L Barts & 1.0 & 288 & 33.3 & 25.7 & 39.2 & 1.4 & 0.3 \\
\hline L Guys & 3.8 & 125 & 50.4 & 11.2 & 30.4 & 1.6 & 6.4 \\
\hline L Kings & 0.0 & 162 & 54.3 & 8.0 & 30.2 & 2.5 & 4.9 \\
\hline L Rfree & 13.6 & 197 & 39.6 & 21.3 & 26.4 & 1.0 & 11.7 \\
\hline L St.G & 14.5 & 65 & 46.2 & 23.1 & 23.1 & & 7.7 \\
\hline L West & 0.0 & 303 & 39.3 & 40.6 & 18.5 & 1.7 & \\
\hline Leeds & 0.0 & 184 & 79.9 & 13.0 & 4.9 & 1.1 & 1.1 \\
\hline Leic & 7.6 & 269 & 75.8 & 19.0 & 3.0 & 1.1 & 1.1 \\
\hline Liv Ain & 4.5 & 63 & 92.1 & 1.6 & 1.6 & 1.6 & 3.2 \\
\hline Liv Roy & 1.1 & 93 & 90.3 & 4.3 & 2.2 & & 3.2 \\
\hline M RI & 7.0 & 186 & 75.8 & 10.2 & 10.8 & & 3.2 \\
\hline Middlbr & 0.0 & 108 & 96.3 & 2.8 & & 0.9 & \\
\hline Newc & 0.0 & 95 & 95.8 & 3.2 & 1.1 & & \\
\hline Norwch & 1.3 & 75 & 100.0 & & & & \\
\hline Nottm & 0.0 & 113 & 89.4 & 6.2 & 2.7 & & 1.8 \\
\hline Oxford & 1.2 & 164 & 82.9 & 9.1 & 5.5 & 1.2 & 1.2 \\
\hline Plymth & 0.0 & 63 & 98.4 & 1.6 & & & \\
\hline Ports & 6.6 & 185 & 96.8 & 2.2 & 0.5 & & 0.5 \\
\hline Prestn & 1.3 & 149 & 86.6 & 12.8 & 0.7 & & \\
\hline Redng & 14.5 & 100 & 80.0 & 12.0 & 6.0 & 1.0 & 1.0 \\
\hline Salford & 0.9 & 110 & 76.4 & 20.9 & 1.8 & & 0.9 \\
\hline Sheff & 4.4 & 131 & 88.6 & 6.9 & 2.3 & & 2.3 \\
\hline Shrew & 0.0 & 61 & 91.8 & 6.6 & 1.6 & & \\
\hline Stevng & 4.4 & 152 & 72.4 & 16.4 & 7.9 & 0.7 & 2.6 \\
\hline Sthend & 16.7 & 35 & 97.1 & & & & 2.9 \\
\hline Stoke & 8.0 & 92 & 93.5 & 3.3 & 2.2 & & 1.1 \\
\hline Sund & 0.0 & 49 & 98.0 & 2.0 & & & \\
\hline Truro & 0.0 & 46 & 100.0 & & & & \\
\hline Wirral & 2.9 & 66 & 98.5 & & & 1.5 & \\
\hline Wolve & 1.1 & 87 & 69.0 & 21.8 & 5.7 & 2.3 & 1.1 \\
\hline York & 0.0 & 36 & 94.4 & & 2.8 & & 2.8 \\
\hline
\end{tabular}


Table 1.7. Continued

\begin{tabular}{|c|c|c|c|c|c|c|c|}
\hline Centre & $\begin{array}{c}\% \text { data not } \\
\text { available }\end{array}$ & $\begin{array}{c}N \text { with } \\
\text { data }\end{array}$ & \multicolumn{5}{|c|}{ Percentage in each ethnic group } \\
\hline Antrim & 0.0 & 29 & 96.6 & & 3.4 & & \\
\hline Belfast & 1.4 & 68 & 98.5 & & 1.5 & & \\
\hline Newry & 0.0 & 23 & 100.0 & & & & \\
\hline \multicolumn{8}{|l|}{ Wales } \\
\hline Bangor & 0.0 & 24 & 100.0 & & & & \\
\hline Cardff & 0.0 & 169 & 89.3 & 7.1 & 2.4 & 0.6 & 0.6 \\
\hline Clwyd & 7.1 & 13 & 100.0 & & & & \\
\hline Swanse & 0.9 & 109 & 100.0 & & & & \\
\hline Wrexm & 2.7 & 36 & 97.2 & & & 2.8 & \\
\hline
\end{tabular}

Blank cells - no reported patients

(9.5\% from 6.3\%) and was similar in under and over 65 year olds (9.3\% and $9.7 \%$ respectively). Five centres had missing PRD for more than 25\% of incident patients and for these centres the percentages in the diagnostic categories are not shown in table 1.8.

The UKRR continues to be concerned about centres with apparently very high data completeness for PRD but also very high rates of 'uncertain' diagnoses (EDTA code 00: Chronic renal failure; aetiology uncertain). It is accepted that there will inevitably be a number of patients with uncertain aetiology and that the proportion of these patients will vary between clinicians and centres as the definitions of e.g. renal vascular disease and hypertensive renal disease remain relatively subjective. There was again a lot of variability between centres but, as in previous years, a small number of centres had far higher percentages with 'uncertain' diagnosis than other centres. This year, there were three centres with diagnosis 'uncertain' for over $45 \%$ of their incident patients - Cambridge (50\%), Colchester (67\%) and Ipswich (49\%). As the numbers with the specific PRDs are likely to be falsely low in these centres, the breakdown into these categories has not been shown in table 1.8 or been used in the country and UK averages. These centres have also been excluded where PRD is used to stratify analyses.

As in previous years, there was a lot of variability between centres in the percentages with the specific diagnoses (partly due to the reasons mentioned above). For example, the percentage with diabetes as PRD varied from about $8 \%$ to over $46 \%$ of incident patients. The overall percentage with uncertain aetiology continued to decrease (14.5\% for 2013 versus $15.9 \%$ for 2012 and $17.3 \%$ for 2011$)$.

The overall UK distribution of PRDs is shown in table 1.9. Diabetic nephropathy was the most common renal diagnosis in both the under and over 65 year age groups, accounting for $25 \%$ of all (non-missing) incident diagnoses. Glomerulonephritis and autosomal dominant polycystic kidney disease (ADPKD) made up higher proportions of the younger than the older incident cohorts ( $18 \%$ vs. $11 \%$ and $11 \%$ vs. $4 \%$ respectively), whilst patients with renal vascular disease comprised a much higher percentage of the older rather than the younger patients ( $10 \%$ vs. $1 \%)$. Uncertainty about the underlying diagnosis was also much more likely in the older rather than the younger cohort (18\% vs. $11 \%)$.

For all primary renal diagnoses except ADPKD, the male to female ratio was 1.3 or greater. This gender difference may relate to factors such as smoking, hypertension, atheroma and renal vascular disease, which are more common in males and may influence the rate of progression of renal failure.

Table 1.10 shows the incidence rates for each PRD per million population for the 2013 cohort. The incidence of RRT due to diabetes as PRD was somewhat higher in Wales than in the other countries. As there were some 
Table 1.8. Distribution of primary renal diagnosis by centre in the 2013 incident RRT cohort

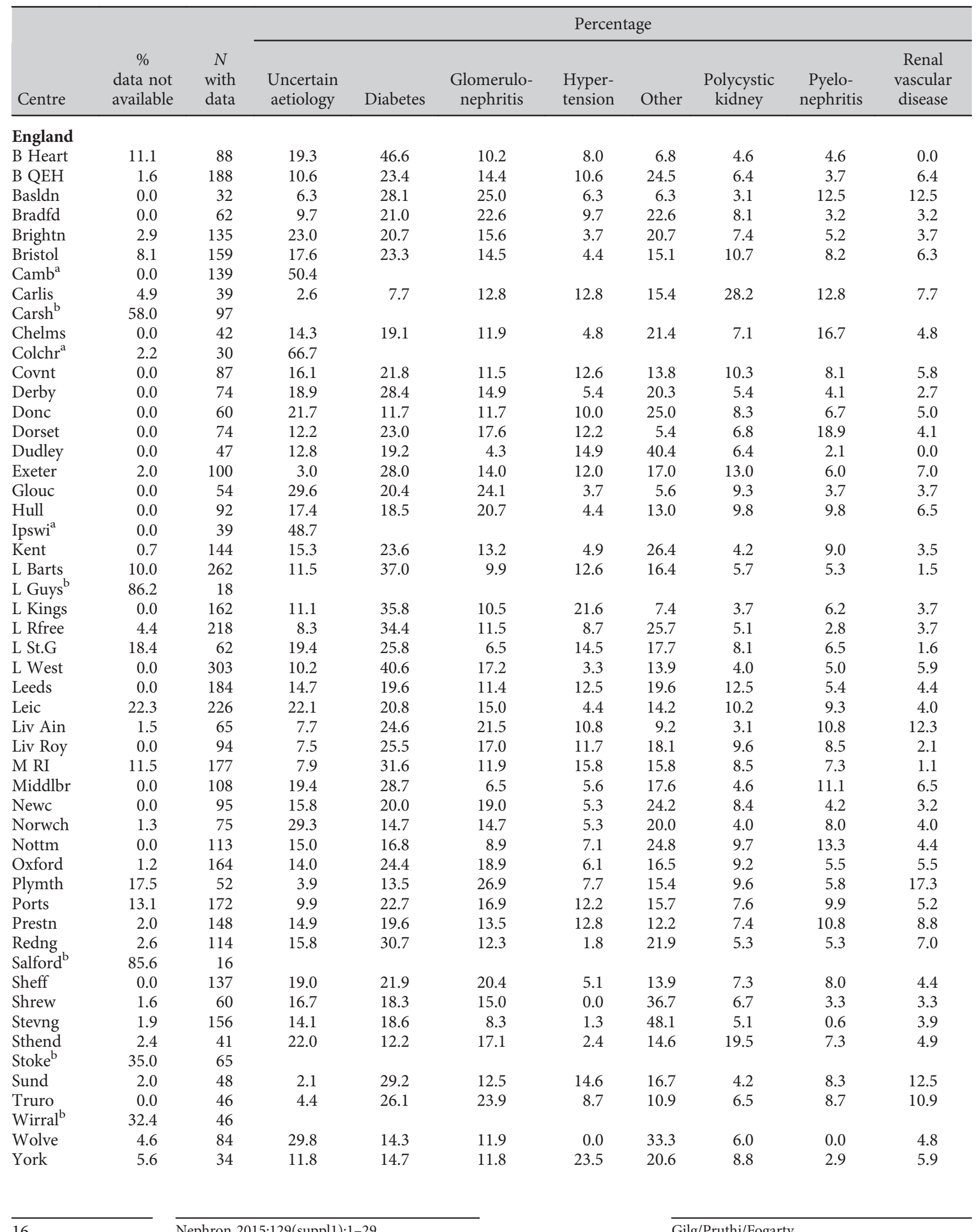


Table 1.8. Continued

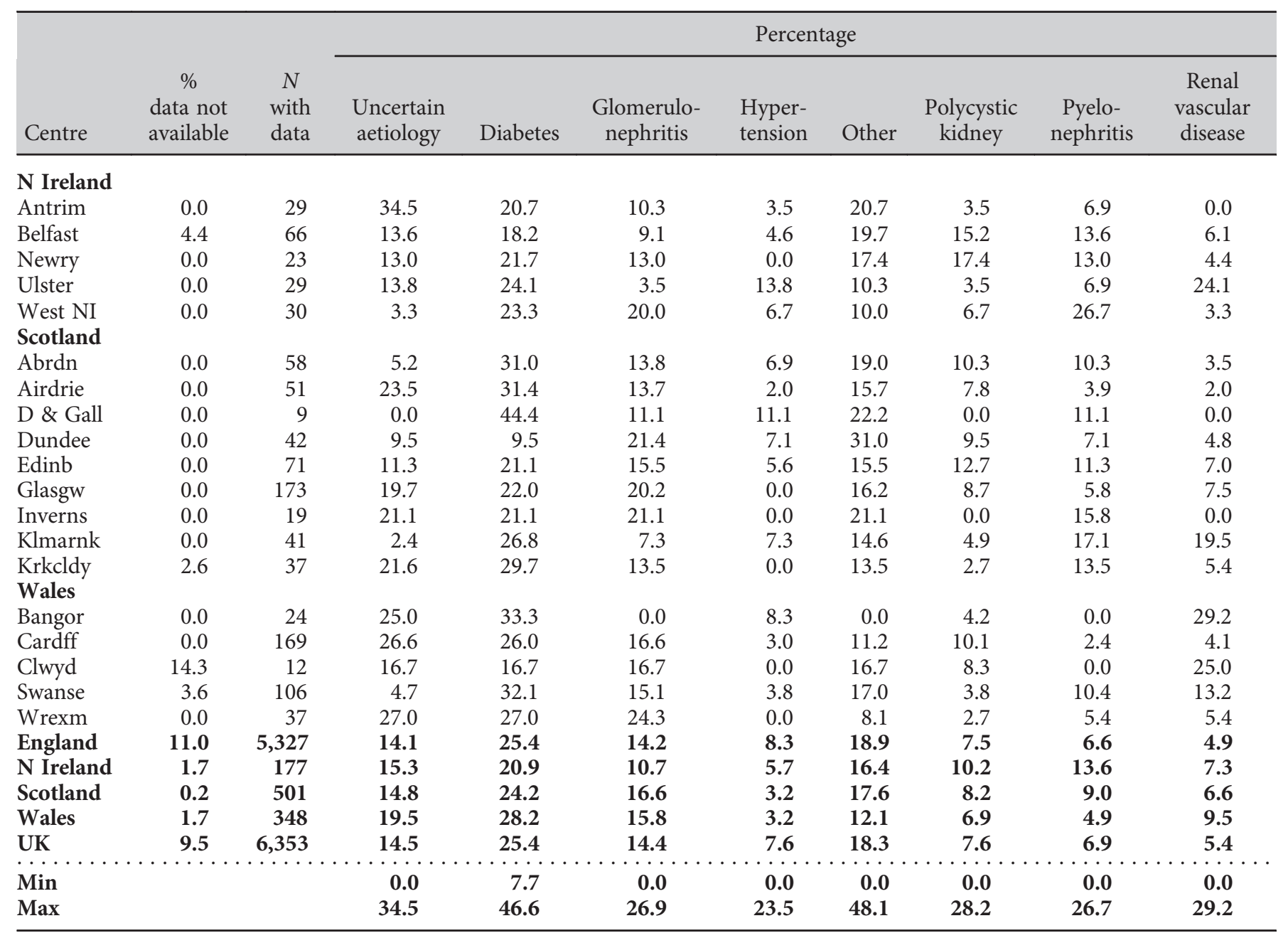

The percentage in each category has been calculated after excluding those patients with data not available

${ }^{a}$ For those centres judged to have high \% uncertain aetiology, the percentages in the other diagnostic categories have not been calculated and these centres have not been included in the country and UK averages or the $\mathrm{min} / \mathrm{max}$ values

${ }^{\mathrm{b}}$ For those centres with $>25 \%$ missing primary diagnoses, the percentages in the diagnostic categories have not been calculated

Table 1.9. Percentage distribution of primary renal diagnosis by age in the 2013 incident RRT cohort

\begin{tabular}{lccc}
\hline & \multicolumn{3}{c}{ Percentage with diagnosis } \\
\cline { 2 - 4 } Diagnosis & Age $<65$ & Age $\geqslant 65$ & All patients \\
\hline Diabetes & 27.1 & 23.5 & 25.4 \\
Glomerulonephritis & 17.8 & 10.9 & 14.4 \\
Pyelonephritis & 7.7 & 6.1 & 6.9 \\
Hypertension & 6.4 & 8.8 & 7.6 \\
Polycystic kidney & 11.2 & 3.7 & 7.6 \\
Renal vascular disease & 1.3 & 9.7 & 5.4 \\
Other & 17.5 & 19.1 & 18.3 \\
Uncertain aetiology & 10.9 & 18.3 & 14.5 \\
\hline
\end{tabular}

Percentages calculated after excluding those patients with data not available missing data, the rates for at least some of the diagnoses will be underestimates.

\section{First established treatment modality}

In 2013, the first treatment recorded, irrespective of any later change, was haemodialysis in $72.0 \%$ of patients, peritoneal dialysis in $19.4 \%$ and pre-emptive transplant in $8.6 \%$. The previous year on year fall seen in the proportion of patients starting on PD levelled off during the last six years (table 1.11). The percentage having a pre-emptive transplant has continued to rise (up by 65\% from 2008). Table F.1.3 in appendix F: Additional Data Tables for $2013 \mathrm{New}$ and Existing Patients gives the treatment breakdown at start of RRT by centre. 
Table 1.10. Primary renal diagnosis RRT incidence rates (2013) per million population (unadjusted)

\begin{tabular}{|c|c|c|c|c|c|}
\hline Diagnosis & England & $\mathrm{N}$ Ireland & Scotland & Wales & UK \\
\hline Diabetes & 25.2 & 20.2 & 22.7 & 31.8 & 25.1 \\
\hline Glomerulonephritis & 14.1 & 10.4 & 15.6 & 17.8 & 14.3 \\
\hline Hypertension & 8.3 & 5.5 & 3.0 & 3.6 & 7.5 \\
\hline Polycystic kidney & 7.4 & 9.8 & 7.7 & 7.8 & 7.5 \\
\hline Renal vascular disease & 4.9 & 7.1 & 6.2 & 10.7 & 5.3 \\
\hline Data not available & 12.3 & 1.6 & 0.2 & 1.9 & 10.4 \\
\hline All & 111 & 98 & 94 & 115 & 110 \\
\hline
\end{tabular}

The overall rates per country may be slightly different to those in table 1.2 as those centres whose PRD data has not been used have been excluded from both the numerator and the denominator here

Many patients undergo a brief period of HD before switches to other modalities are, or can be, considered. Therefore, the established modality at 90 days is more representative of the elective first modality and this modality was used for the remainder of this section. For these analyses, the incident cohort from 1st October 2012 to 30th September 2013 was used so that follow up to 90 days was possible for all patients. By 90 days, 5.1\% of incident patients had died and a further $0.2 \%$ had stopped treatment, leaving $94.7 \%$ of the original cohort still on RRT. Table 1.12 shows the percentages on each treatment modality at 90 days both as percentages of all of those starting RRT and then of those still on treatment at 90 days. Expressed as percentages of the whole incident cohort, $66.1 \%$ were on HD at 90 days, $19.0 \%$ were on PD

Table 1.11. Treatment at start and at 90 days by year of start

\begin{tabular}{lccc}
\hline Start & $\begin{array}{c}\text { HD } \\
(\%)\end{array}$ & $\begin{array}{c}\text { PD } \\
(\%)\end{array}$ & $\begin{array}{c}\text { Transplant } \\
(\%)\end{array}$ \\
\hline Day 0 treatment & & & \\
2008 & 75.4 & 19.4 & 5.2 \\
2009 & 76.3 & 18.0 & 5.7 \\
2010 & 74.6 & 18.6 & 6.8 \\
2011 & 72.7 & 20.4 & 6.9 \\
2012 & 72.9 & 19.6 & 7.5 \\
2013 & 72.0 & 19.4 & 8.6 \\
Day 90 treatment & & & \\
Oct 2007 to end Sept 2008 & 72.2 & 21.6 & 6.2 \\
Oct 2008 to end Sept 2009 & 73.9 & 19.2 & 6.9 \\
Oct 2009 to end Sept 2010 & 72.6 & 19.4 & 8.0 \\
Oct 2010 to end Sept 2011 & 70.8 & 20.6 & 8.7 \\
Oct 2011 to end Sept 2012 & 70.8 & 20.3 & 9.0 \\
Oct 2012 to end Sept 2013 & 69.8 & 20.1 & 10.1 \\
\hline
\end{tabular}

and $9.5 \%$ had received a transplant. Expressed as percentages of those still receiving RRT at 90 days, $69.8 \%$ were on $\mathrm{HD}, 20.1 \%$ on PD and $10.1 \%$ had received a transplant.

Figure 1.8 shows the modality breakdown with the HD patients further subdivided. Of those still on RRT at 90 days, 39\% were treated with hospital HD, 31\% with satellite $\mathrm{HD}$, and only $0.2 \%$ were receiving home $\mathrm{HD}$ at this early stage. This $0.2 \%$ on home HD was only 13 patients (split between seven centres). Chapter 2 UK Renal Replacement Therapy Prevalence in 2013 shows that prevalent numbers of home HD patients have grown to $4.1 \%$ of all dialysis patients.

The percentage of incident patients who had died by 90 days varied considerably between centres $(0 \%$ to $20 \%)$. Differences in the definition of whether patients have acute or chronic renal failure may be a factor in this apparent variation along with possible differences in clinical practice.

The percentage of patients still on RRT at 90 days who had a functioning transplant at 90 days varied between centres from $0 \%$ to $31 \%$ (between $7 \%$ and $31 \%$ for transplanting centres and between $0 \%$ and $12 \%$ for nontransplanting centres). The mean percentage of the incident cohort with a functioning transplant at 90 days was greater in transplanting compared to non-transplanting centres (13.4\% vs. 6.0\%). One possible reason could be that some patients transplanted pre-emptively were attributed to the incident cohort of the transplanting centre rather than that of the referring centre.

Table 1.13 gives the HD/PD breakdown for those incident patients on dialysis at 90 days. The breakdown is given by age group and overall. The percentage on PD at 90 days was about 65\% higher in patients aged 
Table 1.12. RRT modality at 90 days by centre (incident cohort $1 / 10 / 2012$ to $30 / 09 / 2013$ )

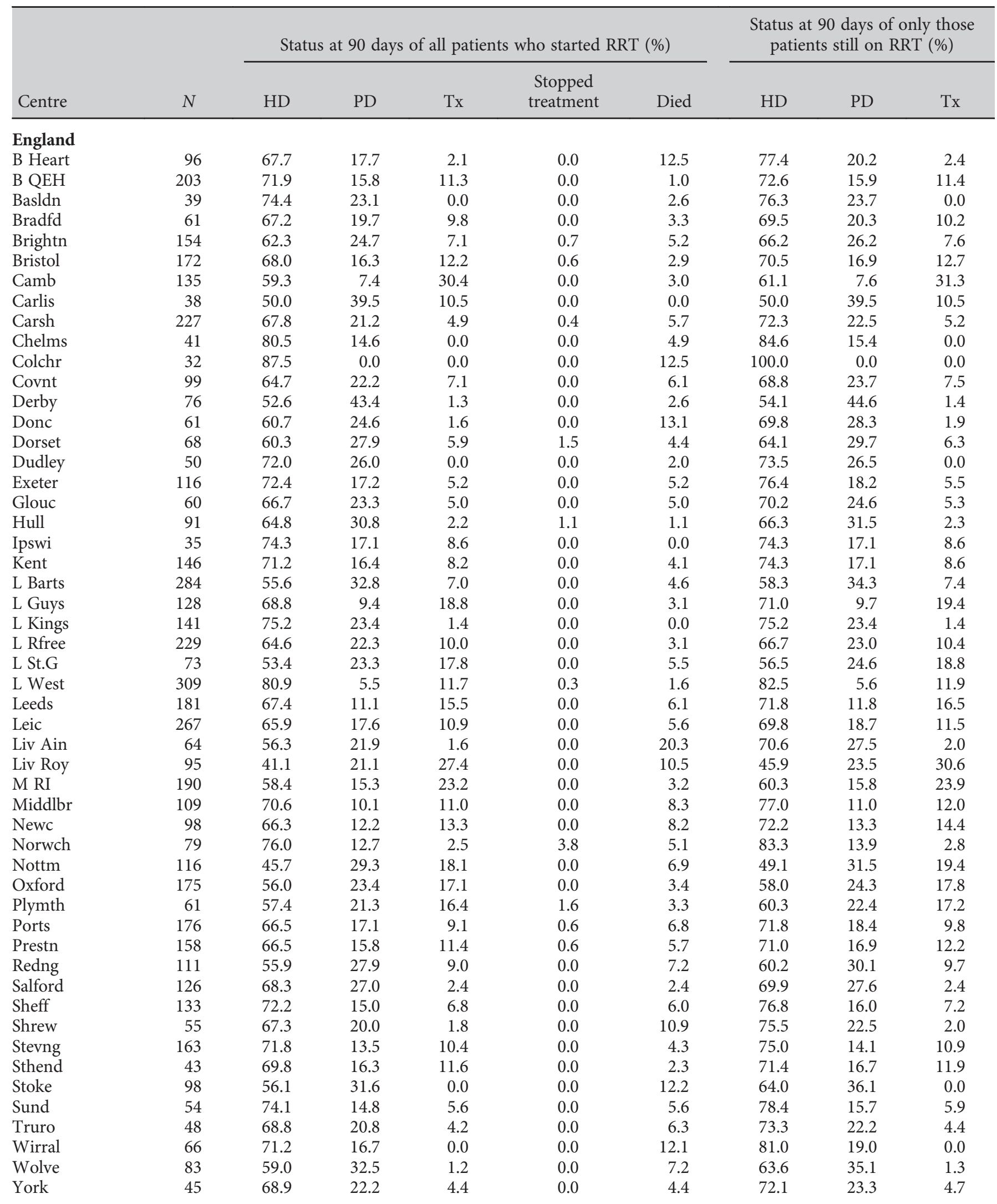


Table 1.12. Continued

\begin{tabular}{|c|c|c|c|c|c|c|c|c|c|}
\hline \multirow[b]{2}{*}{ Centre } & \multirow[b]{2}{*}{$N$} & \multicolumn{5}{|c|}{ Status at 90 days of all patients who started RRT (\%) } & \multicolumn{3}{|c|}{$\begin{array}{l}\text { Status at } 90 \text { days of only those } \\
\text { patients still on RRT (\%) }\end{array}$} \\
\hline & & HD & $\mathrm{PD}$ & $\mathrm{Tx}$ & $\begin{array}{l}\text { Stopped } \\
\text { treatment }\end{array}$ & Died & HD & $\mathrm{PD}$ & $\mathrm{Tx}$ \\
\hline \multicolumn{10}{|l|}{ N Ireland } \\
\hline Antrim & 30 & 70.0 & 16.7 & 0.0 & 0.0 & 13.3 & 80.8 & 19.2 & 0.0 \\
\hline Ulster & 30 & 70.0 & 6.7 & 0.0 & 6.7 & 16.7 & 91.3 & 8.7 & 0.0 \\
\hline West NI & 29 & 51.7 & 31.0 & 10.3 & 3.5 & 3.5 & 55.6 & 33.3 & 11.1 \\
\hline \multicolumn{10}{|l|}{ Scotland } \\
\hline Abrdn & 56 & 64.3 & 23.2 & 3.6 & 0.0 & 8.9 & 70.6 & 25.5 & 3.9 \\
\hline Airdrie & 55 & 85.5 & 14.6 & 0.0 & 0.0 & 0.0 & 85.5 & 14.6 & 0.0 \\
\hline D \& Gall & 6 & 83.3 & 16.7 & 0.0 & 0.0 & 0.0 & 83.3 & 16.7 & 0.0 \\
\hline Klmarnk & 39 & 71.8 & 18.0 & 5.1 & 0.0 & 5.1 & 75.7 & 18.9 & 5.4 \\
\hline Krkcldy & 39 & 76.9 & 12.8 & 0.0 & 0.0 & 10.3 & 85.7 & 14.3 & 0.0 \\
\hline \multicolumn{10}{|l|}{ Wales } \\
\hline Bangor & 27 & 74.1 & 14.8 & 0.0 & 0.0 & 11.1 & 83.3 & 16.7 & 0.0 \\
\hline Cardff & 167 & 67.1 & 15.0 & 12.6 & 0.0 & 5.4 & 70.9 & 15.8 & 13.3 \\
\hline Clwyd & 16 & 75.0 & 18.8 & 6.3 & 0.0 & 0.0 & 75.0 & 18.8 & 6.3 \\
\hline Swanse & 118 & 63.6 & 21.2 & 5.9 & 0.9 & 8.5 & 70.1 & 23.4 & 6.5 \\
\hline Wrexm & 40 & 70.0 & 20.0 & 5.0 & 0.0 & 5.0 & 73.7 & 21.1 & 5.3 \\
\hline England & 5,958 & 65.5 & 19.5 & 9.7 & 0.2 & 5.1 & 69.2 & 20.6 & 10.3 \\
\hline N Ireland & 188 & 61.2 & 20.2 & 9.6 & 2.1 & 6.9 & 67.3 & 22.2 & 10.5 \\
\hline Scotland & 497 & 73.8 & 14.3 & 8.3 & 0.0 & 3.6 & 76.6 & 14.8 & 8.6 \\
\hline Wales & 368 & 67.1 & 17.7 & 8.4 & 0.3 & 6.5 & 72.0 & 19.0 & 9.0 \\
\hline
\end{tabular}

under 65 years than in older patients (27.8\% vs. $17.1 \%)$. These percentages are similar to those for 2012. In both age groups there was a lot of variability between centres in the percentage on PD.

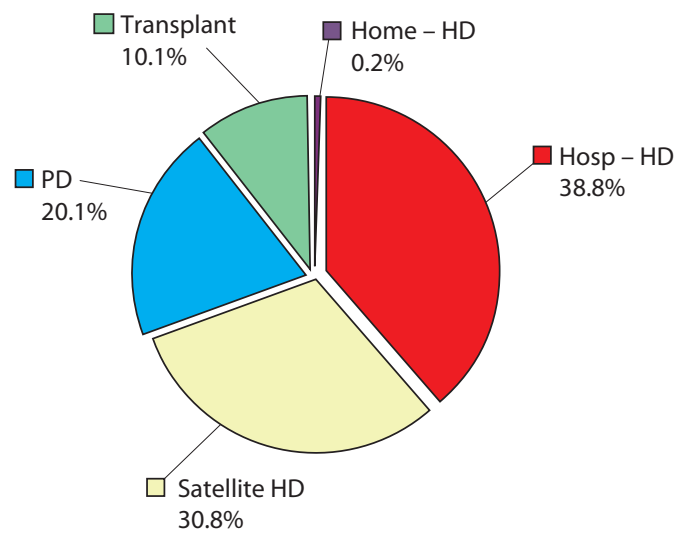

Fig. 1.8. RRT modality at 90 days (incident cohort $1 / 10 / 2012$ to 30/09/2013)
The median age at start for those on $\mathrm{HD}$ at 90 days was 66.7 years compared with 59.3 years for PD. There were eight centres where the percentage of patients treated with PD was the same as or higher in the over $65 \mathrm{~s}$ than the under $65 \mathrm{~s}$ (a similar number to the 10 centres for 2012 and 11 centres for 2011).

\section{Modality change over time}

Table 1.14 gives the breakdown of status/treatment modality at four subsequent time points by initial treatment type for patients starting RRT in 2008. Fifty-one percent of patients who started on HD had died within five years of starting. This compared to $33 \%$ and $5 \%$ for those starting on PD or transplant respectively. Of those patients starting on PD, 91\% were on PD at 90 days but this percentage dropped sharply at the later time points. As expected and in contrast, $92 \%$ of patients starting with a transplant were also transplant patients at the five year time point. 
Table 1.13. Modality split of patients on dialysis at 90 days (incident cohort $1 / 10 / 2012$ to $30 / 09 / 2013$ )

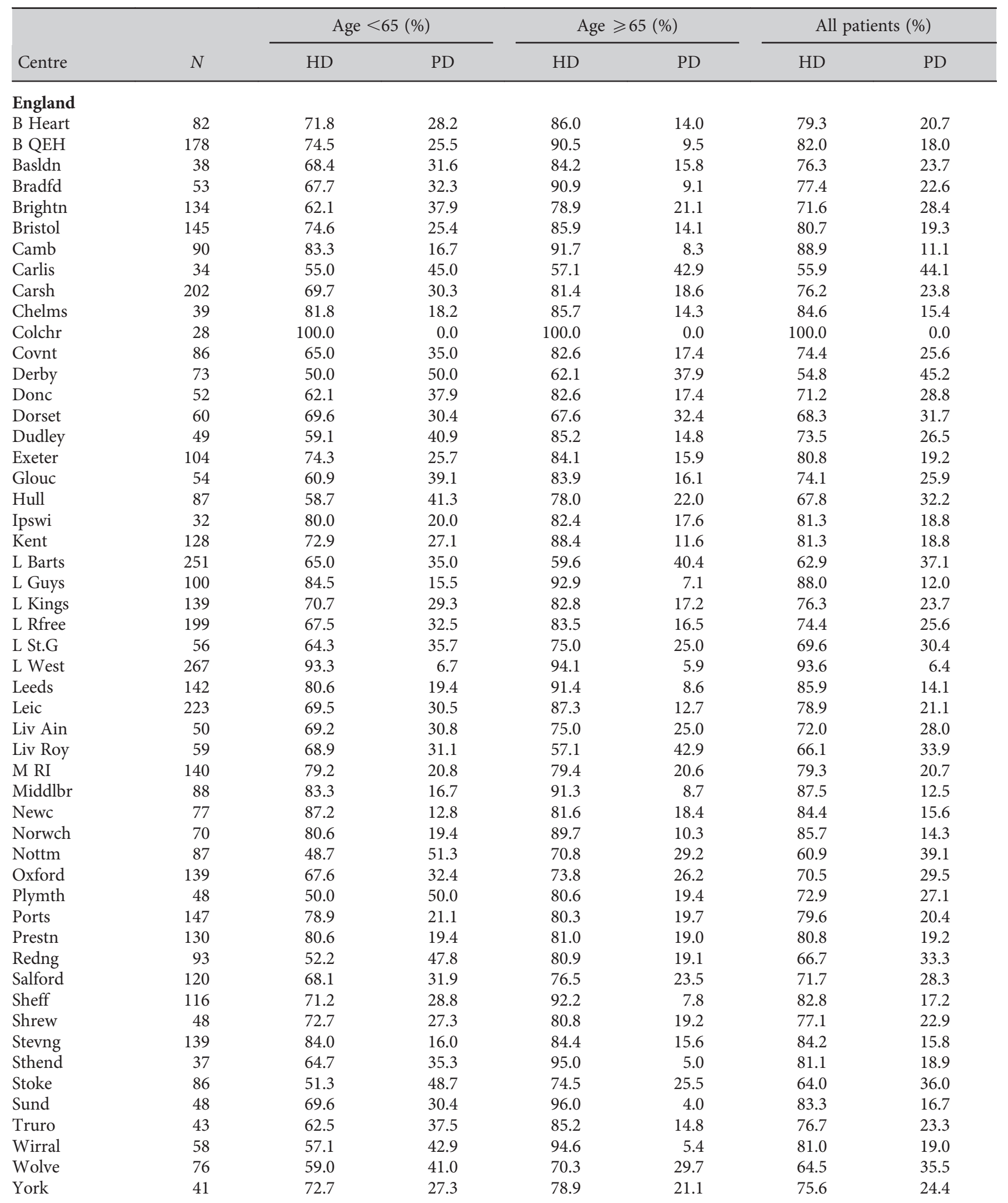


Table 1.13. Continued

\begin{tabular}{|c|c|c|c|c|c|c|c|}
\hline \multirow[b]{2}{*}{ Centre } & \multirow[b]{2}{*}{$N$} & \multicolumn{2}{|c|}{ Age <65 (\%) } & \multicolumn{2}{|c|}{ Age $\geqslant 65(\%)$} & \multicolumn{2}{|c|}{ All patients (\%) } \\
\hline & & HD & $\mathrm{PD}$ & HD & $\mathrm{PD}$ & $\mathrm{HD}$ & PD \\
\hline \multicolumn{8}{|l|}{$\mathrm{N}$ Ireland } \\
\hline Antrim & 26 & 80.0 & 20.0 & 81.3 & 18.8 & 80.8 & 19.2 \\
\hline Belfast & 58 & 78.6 & 21.4 & 80.0 & 20.0 & 79.3 & 20.7 \\
\hline Newry & 22 & 70.0 & 30.0 & 41.7 & 58.3 & 54.5 & 45.5 \\
\hline Ulster & 23 & 80.0 & 20.0 & 94.4 & 5.6 & 91.3 & 8.7 \\
\hline West NI & 24 & 36.4 & 63.6 & 84.6 & 15.4 & 62.5 & 37.5 \\
\hline \multicolumn{8}{|l|}{ Scotland } \\
\hline Abrdn & 49 & 70.8 & 29.2 & 76.0 & 24.0 & 73.5 & 26.5 \\
\hline Airdrie & 55 & 84.0 & 16.0 & 86.7 & 13.3 & 85.5 & 14.5 \\
\hline D \& Gall & 6 & 80.0 & 20.0 & 100.0 & 0.0 & 83.3 & 16.7 \\
\hline Dundee & 37 & 82.4 & 17.6 & 90.0 & 10.0 & 86.5 & 13.5 \\
\hline Edinb & 52 & 92.7 & 7.3 & 90.9 & 9.1 & 92.3 & 7.7 \\
\hline Glasgw & 151 & 87.3 & 12.7 & 86.3 & 13.8 & 86.8 & 13.2 \\
\hline Inverns & 18 & 46.2 & 53.8 & 80.0 & 20.0 & 55.6 & 44.4 \\
\hline Klmarnk & 35 & 87.5 & 12.5 & 73.7 & 26.3 & 80.0 & 20.0 \\
\hline Krkcldy & 35 & 76.9 & 23.1 & 90.9 & 9.1 & 85.7 & 14.3 \\
\hline \multicolumn{8}{|l|}{ Wales } \\
\hline Bangor & 24 & 100.0 & 0.0 & 77.8 & 22.2 & 83.3 & 16.7 \\
\hline Cardff & 137 & 74.2 & 25.8 & 88.0 & 12.0 & 81.8 & 18.2 \\
\hline Clwyd & 15 & 66.7 & 33.3 & 88.9 & 11.1 & 80.0 & 20.0 \\
\hline Swanse & 100 & 60.0 & 40.0 & 83.1 & 16.9 & 75.0 & 25.0 \\
\hline Wrexm & 36 & 57.1 & 42.9 & 90.9 & 9.1 & 77.8 & 22.2 \\
\hline England & 5,065 & 71.4 & 28.6 & 82.7 & 17.3 & 77.1 & 22.9 \\
\hline $\mathrm{N}$ Ireland & 153 & 70.3 & 29.7 & 78.7 & 21.3 & 75.2 & 24.8 \\
\hline Scotland & 438 & 82.7 & 17.3 & 85.0 & 15.0 & 83.8 & 16.2 \\
\hline Wales & 312 & 69.1 & 30.9 & 85.7 & 14.3 & 79.2 & 20.8 \\
\hline UK & 5,968 & 72.2 & 27.8 & 82.9 & 17.1 & 77.6 & 22.4 \\
\hline
\end{tabular}

Table 1.14. Initial and subsequent modalities for patients starting RRT in 2008

\begin{tabular}{|c|c|c|c|c|c|c|}
\hline \multirow[b]{2}{*}{ First treatment } & \multirow[b]{2}{*}{$N$} & \multirow[b]{2}{*}{ Later modality } & \multicolumn{4}{|c|}{ Percentage } \\
\hline & & & 90 days & 1 year & 3 years & 5 years \\
\hline \multirow[t]{5}{*}{ HD } & \multirow[t]{5}{*}{5,034} & HD & 88 & 72 & 48 & 30 \\
\hline & & $\mathrm{PD}$ & 2 & 3 & 2 & 1 \\
\hline & & Transplant & 1 & 4 & 12 & 16 \\
\hline & & Other* & 0 & 1 & 2 & 1 \\
\hline & & Died & 8 & 19 & 38 & 51 \\
\hline \multirow[t]{5}{*}{ PD } & \multirow[t]{5}{*}{1,297} & HD & 6 & 15 & 21 & 18 \\
\hline & & PD & 91 & 69 & 30 & 11 \\
\hline & & Transplant & 1 & 9 & 28 & 37 \\
\hline & & Other* & 0 & 1 & 1 & 1 \\
\hline & & Died & 2 & 7 & 21 & 33 \\
\hline \multirow[t]{4}{*}{ Transplant } & \multirow[t]{4}{*}{349} & HD & 1 & 1 & 3 & 4 \\
\hline & & PD & 0 & 0 & 0 & 0 \\
\hline & & Transplant & 99 & 97 & 94 & 92 \\
\hline & & Died & 0 & 1 & 3 & 5 \\
\hline
\end{tabular}

*Other e.g. stopped treatment 


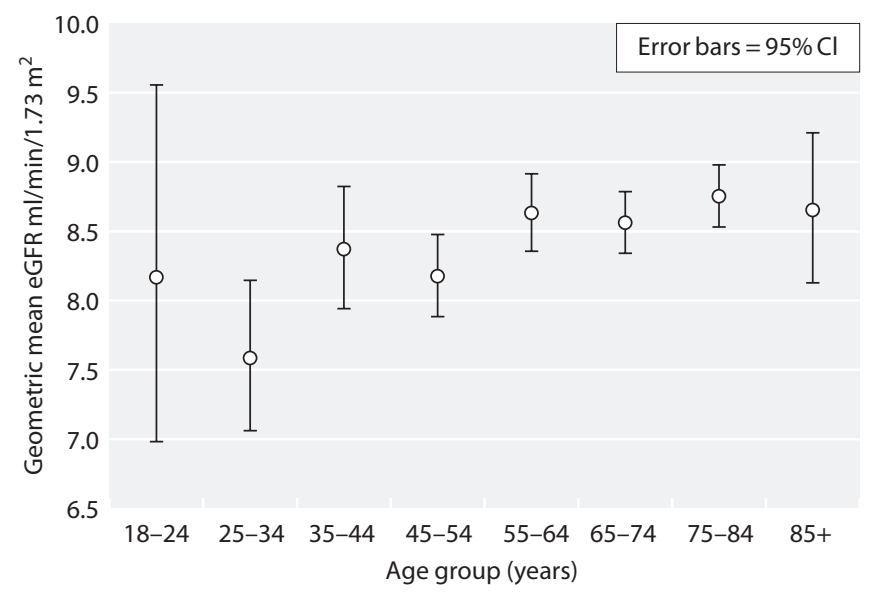

Fig. 1.9. Geometric mean eGFR at start of RRT (2013) by age group

Renal function at the time of starting $R R T$

The mean eGFR at initiation of RRT in 2013 was $8.5 \mathrm{ml} / \mathrm{min} / 1.73 \mathrm{~m}^{2}$. This is shown by age group in figure 1.9.

Figure 1.10 shows serial data from centres reporting annually to the UKRR since 2004. For the six years before 2011 there was higher average eGFR at start of RRT for PD than HD patients but the values were more similar between treatments for 2011 to 2013 .

Some caution should be applied to the analyses of eGFR at the start of RRT as data was only available for less than half of the incident patients (approximately 3,000 for 2013) and almost half of these came from only 10 centres. Three-quarters of the values came from 22 centres. Further caution should be applied as a review of pre-RRT biochemistry in nine renal centres revealed that up to $18 \%$ of patients may have had an incorrect date of starting RRT allocated and thus, the eGFR used

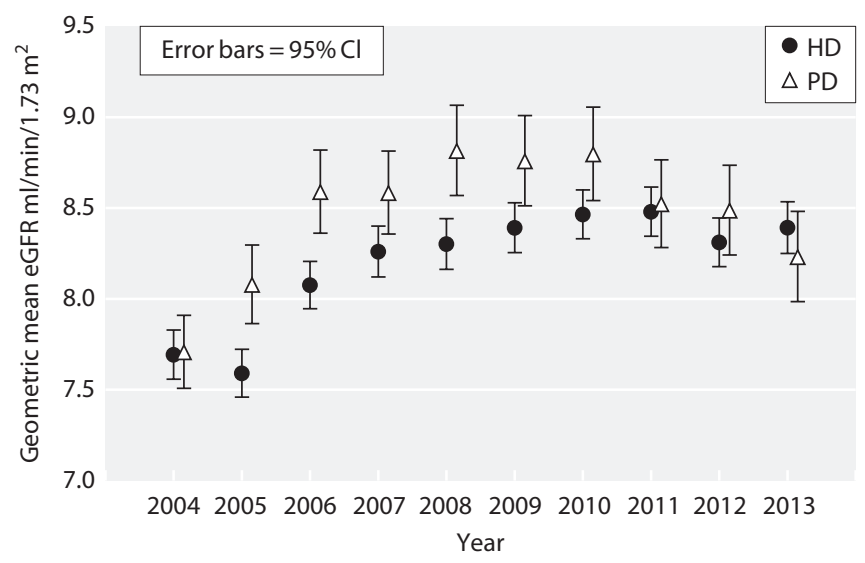

Fig. 1.10. eGFR on starting RRT 2004 to 2013, PD and HD (restricted to centres reporting since 2004) for analysis may have been taken whilst they were already receiving RRT. For details see the 12th Annual Report chapter 13: The UK Renal Registry Advanced CKD Study 2009 [4]. In the future the UKRR hopes to address this and related timeline anomalies by more frequent data downloads.

\section{Late presentation and delayed referral of incident patients}

\section{Introduction}

Late presentation to a nephrologist is regarded as a negative aspect in renal care. It can be defined in a number of ways as it has a range of possible causes. There are many patients with chronic kidney disease who are regularly monitored in primary or secondary care and whose referral to nephrology services is delayed (delayed or late referral). In contrast, other patients present late to medical services due to no particular deficiency in the service; those with either such slowly progressive disease as to have remained asymptomatic for many years or the opposite - those with rapidly progressive CKD. The main analyses presented here do not differentiate between these groups and include any patient first seen by renal services within 90 days of starting RRT as 'late presentation'. One analysis attempts to capture 'late referrals': it shows the percentage presenting within 90 days of starting RRT after excluding an acute renal disease group.

\section{Methods}

Date first seen by a nephrologist has not been collected from the Scottish Renal Registry and so Scottish centres were excluded from these analyses. Data were included from all incident patients in English, Welsh or Northern Irish centres in the years 2012 to 2013. This two year cohort was used for most of the analyses in order to make the late presentation percentages more reliably estimated and to allow these to be shown for subgroups of patients. The date first seen in a renal centre and the date of starting RRT were used to define the late presenting cohort. A small amount of data were excluded because of actual or potential inconsistencies. Only data from those centres with $75 \%$ or more completeness for the relevant year were used. Data were excluded if $10 \%$ or more of the patients were reported to have started RRT on the same date as the first presentation. This was because investigation has shown that this is likely due to misunderstanding on the part of the renal centres resulting in incorrect recording of data. After these exclusions, data on 10,502 patients were available for analysis. Presentation times of 90 days or more before start were defined as early presentation and times of less than 90 days were defined as late presentation.

The 'acute' group was made up of those people with conditions likely to present with rapidly deteriorating renal function: crescentic glomerulonephritis (type I, II, III), renal vascular disease due to 
malignant hypertension, renal vascular disease due to polyarteritis, Wegener's granulomatosis, cryoglobulinemic glomerulonephritis, myelomatosis/light chain deposit disease, Goodpasture's syndrome, systemic sclerosis, haemolytic ureaemic syndrome, multi-system disease - other, tubular necrosis (irreversible) or cortical necrosis, kidney tumour(s) and surgical loss of kidney(s).

\section{Results}

Table 1.15 shows the percentage completeness of data for 2012 and 2013. The overall average completeness was over $85 \%$.

\section{Late presentation by centre}

Figure 1.11 shows that late presentation varied between centres from $6 \%$ to $36 \%$ in patients starting
RRT in 2012 to 2013. The overall rate of late presentation was $18.6 \%$ and was $14.0 \%$ once those people with diseases likely to present acutely were excluded. Table 1.16 shows the overall percentage presenting late for the combined 2012/2013 incident cohort, the percentages presenting late amongst those patients defined as not having an 'acute diagnosis' and the percentages amongst nondiabetics (as PRD). The table also shows the percentages presenting less than a year before RRT initiation.

\section{Late presentation in 2013 and the trend over time}

There has been a steady decline nationally in the proportion of patients presenting late to renal services, with some centres achieving $<10 \%$ late presentation rates.

Table 1.15. Percentage completeness of time of presentation data

\begin{tabular}{|c|c|c|c|c|}
\hline \multirow[b]{2}{*}{ Centre } & \multicolumn{2}{|c|}{$N$} & \multicolumn{2}{|c|}{ Percentage completeness } \\
\hline & 2012 & 2013 & 2012 & 2013 \\
\hline \multicolumn{5}{|l|}{ England } \\
\hline B Heart & 102 & 99 & 97.1 & 93.9 \\
\hline B QEH & 213 & 191 & 100.0 & 99.5 \\
\hline Basldn & 53 & 32 & 98.1 & 100.0 \\
\hline Bradfd & 69 & 62 & 97.1 & 100.0 \\
\hline Brightn & 135 & 139 & 91.7 & 98.5 \\
\hline Bristol & 148 & 173 & 96.6 & 49.7 \\
\hline Camb & 125 & 139 & 100.0 & 88.5 \\
\hline Carlis & 19 & 41 & 94.7 & 100.0 \\
\hline Carsh & 243 & 231 & 88.0 & 68.7 \\
\hline Chelms & 46 & 42 & 97.8 & 100.0 \\
\hline Colchr & 29 & 30 & 100.0 & 100.0 \\
\hline Covnt & 113 & 87 & 99.1 & 97.7 \\
\hline Derby & 79 & 74 & 100.0 & 97.3 \\
\hline Donc & 40 & 60 & 97.5 & 91.7 \\
\hline Dorset & 73 & 74 & 98.6 & 100.0 \\
\hline Dudley & 56 & 47 & 98.2 & 100.0 \\
\hline Exeter & 135 & 102 & 97.0 & 97.1 \\
\hline Glouc & 76 & 54 & 96.0 & 96.2 \\
\hline Hull & 97 & 92 & 97.9 & 96.6 \\
\hline Ipswi & 43 & 39 & 97.7 & 94.9 \\
\hline Kent & 115 & 145 & 100.0 & 100.0 \\
\hline L Barts & 268 & 291 & 1.5 & 1.7 \\
\hline L Guys & 129 & 130 & 22.1 & 54.3 \\
\hline L Kings & 124 & 162 & 98.4 & 98.8 \\
\hline L Rfree & 237 & 228 & 99.2 & 98.7 \\
\hline L St.G & 90 & 76 & 65.6 & 52.6 \\
\hline L West & 355 & 303 & 81.6 & 99.0 \\
\hline Leeds & 154 & 184 & 98.0 & 98.3 \\
\hline Leic & 236 & 291 & 97.0 & 96.6 \\
\hline Liv Ain & 63 & 66 & 100.0 & 97.0 \\
\hline Liv Roy & 104 & 94 & 99.0 & 98.9 \\
\hline M RI & 161 & 200 & 92.5 & 99.0 \\
\hline Middlbr & 120 & 108 & 100.0 & 99.1 \\
\hline Newc & 104 & 95 & 88.5 & 94.7 \\
\hline
\end{tabular}

\begin{tabular}{|c|c|c|c|c|}
\hline \multirow[b]{2}{*}{ Centre } & \multicolumn{2}{|c|}{$N$} & \multicolumn{2}{|c|}{ Percentage completeness } \\
\hline & 2012 & 2013 & 2012 & 2013 \\
\hline Norwch & 74 & 76 & 91.9 & * \\
\hline Nottm & 101 & 113 & 98.0 & 97.3 \\
\hline Oxford & 170 & 166 & 98.2 & 96.4 \\
\hline Plymth & 55 & 63 & 40.0 & 68.3 \\
\hline Ports & 160 & 198 & 96.9 & 86.2 \\
\hline Prestn & 146 & 151 & 95.8 & 99.3 \\
\hline Redng & 73 & 117 & 97.3 & 99.2 \\
\hline Salford & 134 & 111 & 10.6 & 0.9 \\
\hline Sheff & 157 & 137 & 98.7 & 99.2 \\
\hline Shrew & 58 & 61 & 98.3 & 100.0 \\
\hline Stevng & 109 & 159 & 99.1 & 98.7 \\
\hline Sthend & 26 & 42 & 100.0 & 97.6 \\
\hline Stoke & 74 & 100 & 98.7 & 78.0 \\
\hline Sund & 71 & 49 & 98.6 & 93.9 \\
\hline Truro & 49 & 46 & 100.0 & 100.0 \\
\hline Wirral & 44 & 68 & 95.4 & 98.5 \\
\hline Wolve & 87 & 88 & 100.0 & 98.9 \\
\hline York & 53 & 36 & 100.0 & $*$ \\
\hline \multicolumn{5}{|l|}{ N Ireland } \\
\hline Antrim & 26 & 29 & 100.0 & 96.6 \\
\hline Belfast & 93 & 69 & 90.3 & 95.7 \\
\hline Newry & 17 & 23 & 100.0 & 100.0 \\
\hline Ulster & 29 & 29 & 100.0 & 100.0 \\
\hline West NI & 21 & 30 & 100.0 & 100.0 \\
\hline \multicolumn{5}{|l|}{ Wales } \\
\hline Bangor & 21 & 24 & 90.5 & 95.8 \\
\hline Cardff & 171 & 169 & 98.8 & 97.6 \\
\hline Clwyd & 22 & 14 & 100.0 & * \\
\hline Swanse & 119 & 110 & 99.2 & 100.0 \\
\hline Wrexm & 34 & 37 & 97.1 & 100.0 \\
\hline England & 5,795 & 5,962 & 87.0 & 84.4 \\
\hline N Ireland & 186 & 180 & 95.2 & 97.8 \\
\hline Wales & 367 & 354 & 98.4 & 94.6 \\
\hline $\mathrm{E}, \mathrm{W} \& \mathrm{NI}$ & 6,348 & 6,496 & 87.9 & 85.3 \\
\hline
\end{tabular}

\footnotetext{
${ }^{*}$ Data not shown as $>10 \%$ of patients reported as starting RRT on the same date as first presentation
} 


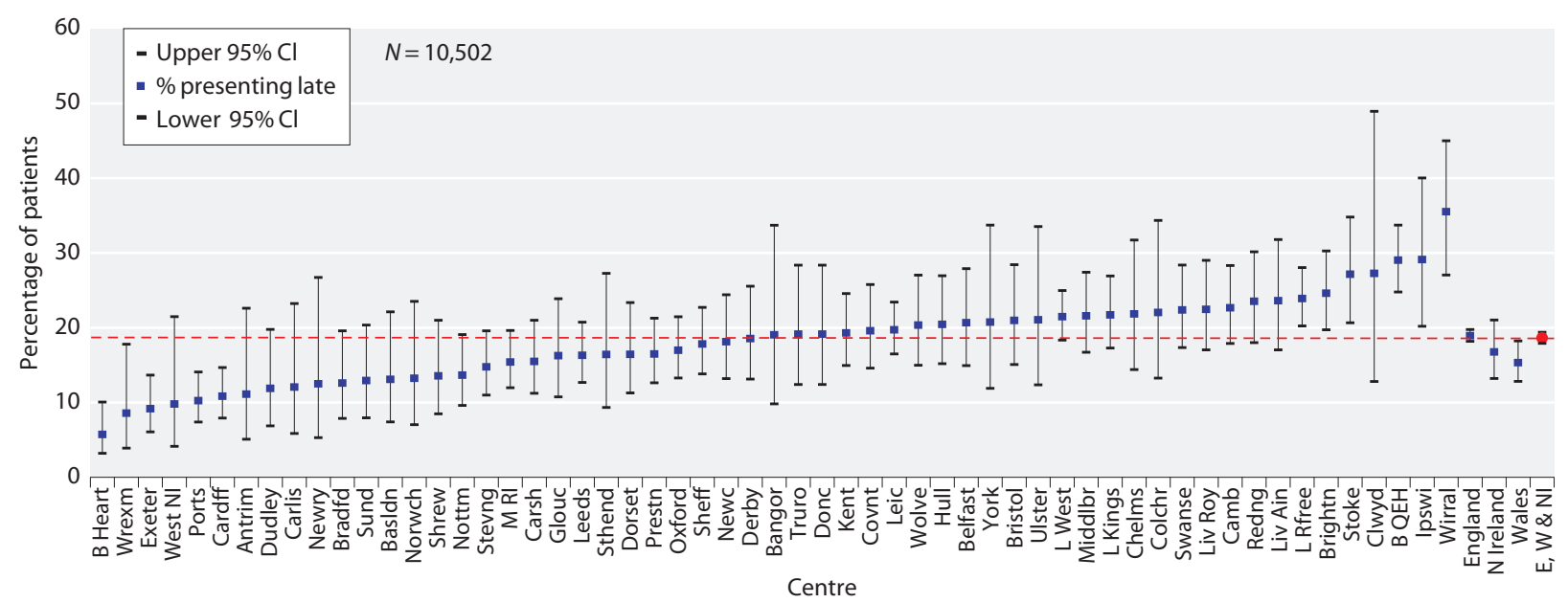

Fig. 1.11. Percentage presenting late (2012/2013)

This may be a consequence of the National CKD guidelines published by the Medical and GP Royal Colleges [5], the Quality and Outcomes Framework (QOF) initiative (www.dh.gov.uk) raising awareness of CKD amongst non-nephrologists and the introduction of estimated GFR reporting.

In 2013, 68.6\% of incident patients presented to nephrology services over a year before they started
RRT. There were $7.8 \%$ of patients presenting within the 6-12 month window before RRT, 5.2\% within 3-6 months and $18.4 \%$ within three months of RRT start. Figure 1.12 shows this breakdown by year for those 26 centres supplying data over $75 \%$ complete for each of the last six years. The figure shows an increase over time in the percentage of patients presenting a year or more before starting RRT. As shown in previous reports this

Table 1.16. Percentage of patients presenting to a nephrologist less than 90 days before RRT initiation and percentage presenting less than a year before initiation (2012/2013 incident patients) by centre

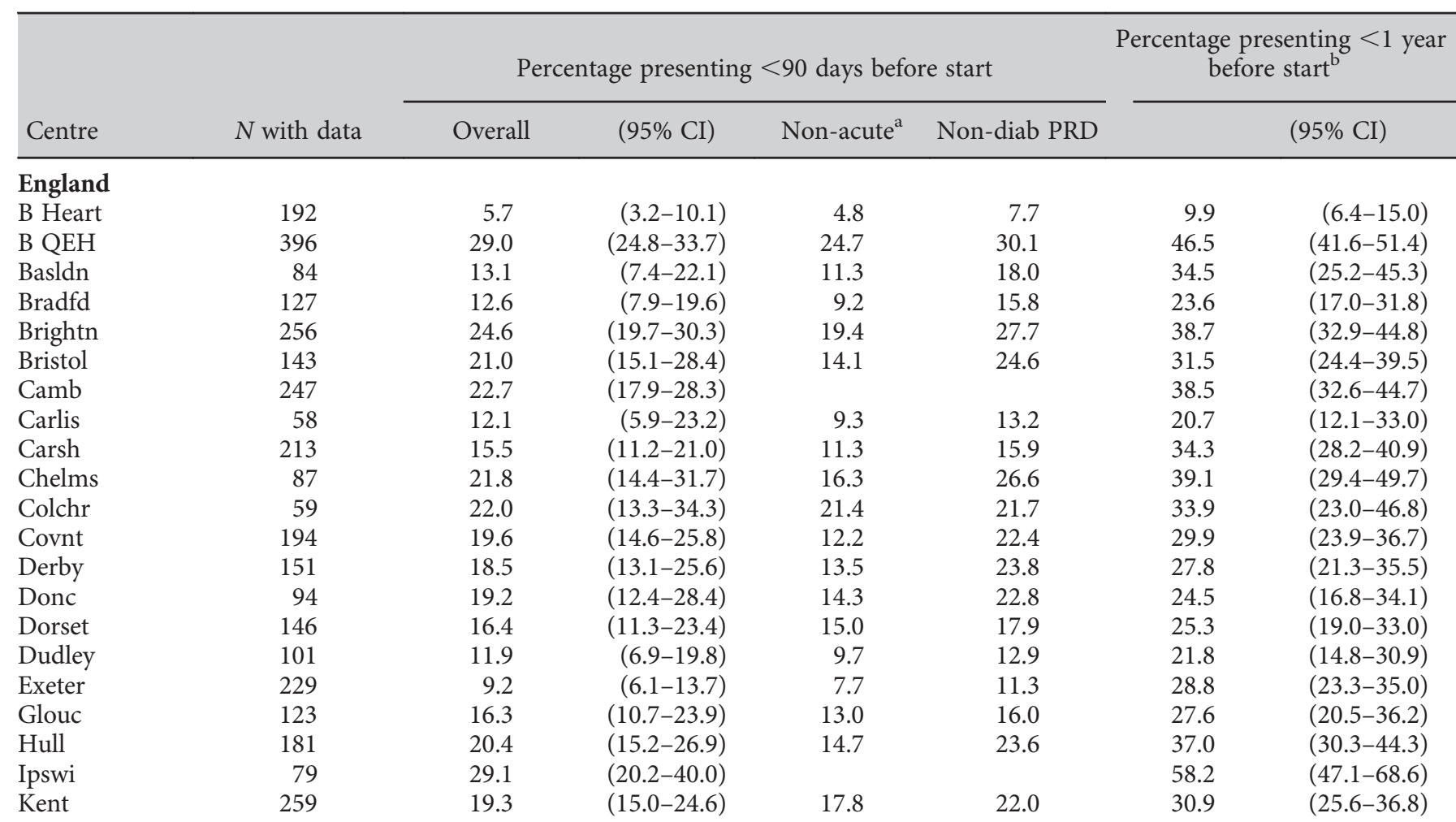


Table 1.16. Continued

\begin{tabular}{|c|c|c|c|c|c|c|c|}
\hline \multirow[b]{2}{*}{ Centre } & \multirow[b]{2}{*}{$N$ with data } & \multicolumn{4}{|c|}{ Percentage presenting $<90$ days before start } & \multicolumn{2}{|c|}{$\begin{array}{c}\text { Percentage presenting }<1 \text { year } \\
\text { before start }\end{array}$} \\
\hline & & Overall & $(95 \% \mathrm{CI})$ & Non-acute ${ }^{a}$ & Non-diab PRD & & $(95 \% \mathrm{CI})$ \\
\hline L Kings & 281 & 21.7 & $(17.3-26.9)$ & 16.5 & 28.2 & 34.2 & $(28.9-39.9)$ \\
\hline L Rfree & 460 & 23.9 & $(20.2-28.0)$ & 20.1 & 27.9 & 44.1 & $(39.7-48.7)$ \\
\hline L West & 587 & 21.5 & $(18.3-25.0)$ & 18.1 & 23.6 & 34.4 & $(30.7-38.4)$ \\
\hline Liv Ain & 127 & 23.6 & $(17.0-31.8)$ & 21.0 & 29.9 & 37.8 & $(29.8-46.5)$ \\
\hline Liv Roy & 187 & 22.5 & $(17.0-29.0)$ & 13.3 & 26.7 & 35.8 & $(29.3-43.0)$ \\
\hline M RI & 344 & 15.4 & $(12.0-19.6)$ & 12.9 & 18.4 & 35.8 & $(30.9-41.0)$ \\
\hline Middlbr & 227 & 21.6 & $(16.7-27.4)$ & 19.1 & 26.4 & 34.4 & $(28.5-40.8)$ \\
\hline Newc & 182 & 18.1 & $(13.2-24.4)$ & 8.7 & 21.5 & 31.3 & $(25.0-38.4)$ \\
\hline Norwch & 68 & 13.2 & $(7.0-23.5)$ & 11.1 & 16.1 & 22.1 & $(13.8-33.4)$ \\
\hline Redng & 187 & 23.5 & $(18.0-30.1)$ & 17.3 & 31.0 & 36.4 & $(29.8-43.5)$ \\
\hline Sheff & 286 & 17.8 & $(13.8-22.7)$ & 13.4 & 22.0 & 27.3 & $(22.4-32.7)$ \\
\hline Shrew & 118 & 13.6 & $(8.5-21.0)$ & 13.5 & 15.2 & 33.9 & $(25.9-42.9)$ \\
\hline Stevng & 264 & 14.8 & $(11.0-19.6)$ & 11.3 & 16.2 & 20.1 & $(15.7-25.3)$ \\
\hline Sthend & 67 & 16.4 & $(9.3-27.3)$ & 14.1 & 19.0 & 28.4 & $(18.9-40.2)$ \\
\hline Stoke & 151 & 27.2 & $(20.7-34.8)$ & 22.2 & 29.1 & 45.0 & $(37.3-53.0)$ \\
\hline Sund & 116 & 12.9 & $(8.0-20.4)$ & 10.8 & 11.8 & 21.6 & $(15.0-30.0)$ \\
\hline Truro & 94 & 19.2 & $(12.4-28.4)$ & 14.3 & 22.1 & 31.9 & $(23.3-42.0)$ \\
\hline Wirral & 107 & 35.5 & $(27.0-45.0)$ & & & 52.3 & $(42.9-61.6)$ \\
\hline Wolve & 172 & 20.4 & $(15.0-27.0)$ & 17.8 & 21.3 & 36.6 & $(29.8-44.1)$ \\
\hline York & 53 & 20.8 & $(11.9-33.7)$ & 15.6 & 21.4 & 28.3 & $(17.8-41.8)$ \\
\hline \multicolumn{8}{|l|}{$\mathrm{N}$ Ireland } \\
\hline Cardff & 332 & 10.8 & $(7.9-14.7)$ & 9.1 & 13.6 & 26.5 & $(22.0-31.5)$ \\
\hline Clwyd & 22 & 27.3 & $(12.8-48.9)$ & 22.2 & 22.2 & 36.4 & $(19.3-57.7)$ \\
\hline Swanse & 219 & 22.4 & $(17.3-28.4)$ & 14.8 & 27.7 & 38.8 & $(32.6-45.4)$ \\
\hline Wrexm & 70 & 8.6 & $(3.9-17.8)$ & 7.6 & 11.5 & 25.7 & $(16.8-37.2)$ \\
\hline England & 9,465 & 18.9 & $(18.2-19.8)$ & 14.3 & 21.3 & 32.2 & $(31.3-33.2)$ \\
\hline N Ireland & 352 & 16.8 & (13.2-21.0) & 12.7 & 19.3 & 29.3 & $(24.7-34.2)$ \\
\hline Wales & 685 & 15.3 & $(12.8-18.2)$ & 11.7 & 18.9 & 30.8 & $(27.5-34.4)$ \\
\hline E, W \& NI & 10,502 & 18.6 & (17.9-19.4) & 14.0 & 21.1 & 32.0 & $(31.2-32.9)$ \\
\hline Min & & 5.7 & & 4.8 & 7.7 & 9.9 & \\
\hline Quartile 1 & & 13.6 & & 10.9 & 16.0 & 25.9 & \\
\hline Quartile 3 & & 21.7 & & 17.1 & 23.9 & 35.8 & \\
\hline $\operatorname{Max}$ & & 35.5 & & 24.7 & 31.0 & 58.2 & \\
\hline
\end{tabular}

Blank cells - data for PRD not used due to high \% with uncertain aetiology

${ }^{a}$ Non-acute group excludes crescentic (extracapillary) glomerulonephritis (type I, II, III), nephropathy (interstitial) due to cis-platinum, renal vascular disease due to malignant hypertension, renal vascular disease due to polyarteritis, Wegener's granulomatosis, cryoglobulinemic glomerulonephritis, myelomatosis/light chain deposit disease, Goodpasture's syndrome, systemic sclerosis (scleroderma), haemolytic ureaemic syndrome, multi-system disease - other, tubular necrosis (irreversible) or cortical necrosis, Balkan nephropathy, kidney tumour(s), and traumatic or surgical loss of kidney(s)

${ }^{\mathrm{b}}$ The remaining patients starting RRT therefore presented over 1 year beforehand 


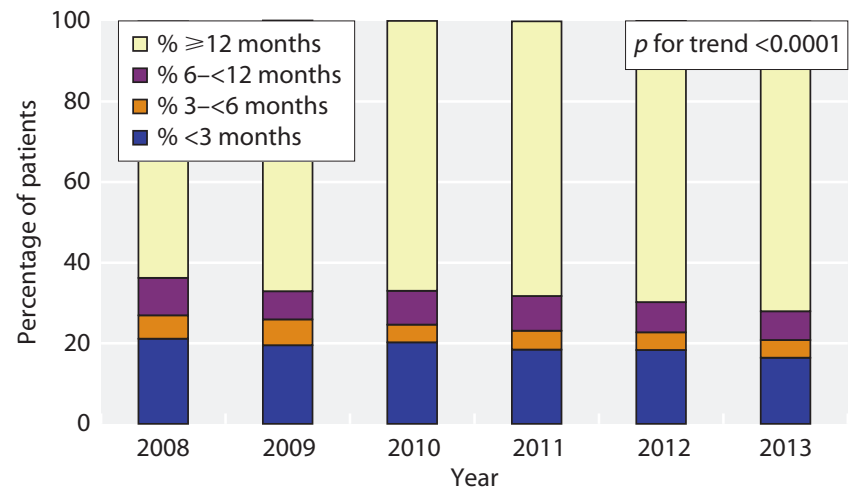

Fig. 1.12. Late presentation rate by year (2008-2013) Restricted to centres reporting continuous data for 2008-2013

increase was most marked in the years just before those shown in the figure. In 2005, only $52.6 \%$ of incident patients presented over a year before they needed to start RRT compared with nearly $70 \%$ in 2013.

\section{Age and late presentation}

In the combined 2012/2013 incident cohort, patients who presented late were not significantly older or younger than patients who presented earlier ( $>90$ days before RRT initiation) (median age 65.4 vs. 64.5 years: $p=0.5$ ). Except for the two youngest age groups, the median duration of pre-RRT care did not vary greatly with age group (figure 1.13).

\section{Gender and late presentation}

In the 2012/2013 cohort, there was no significant difference in the ratio of males to females by time of presentation (male: female ratio 1.70 in early presentation, 1.77 in late presentation, $p=0.4$ ).

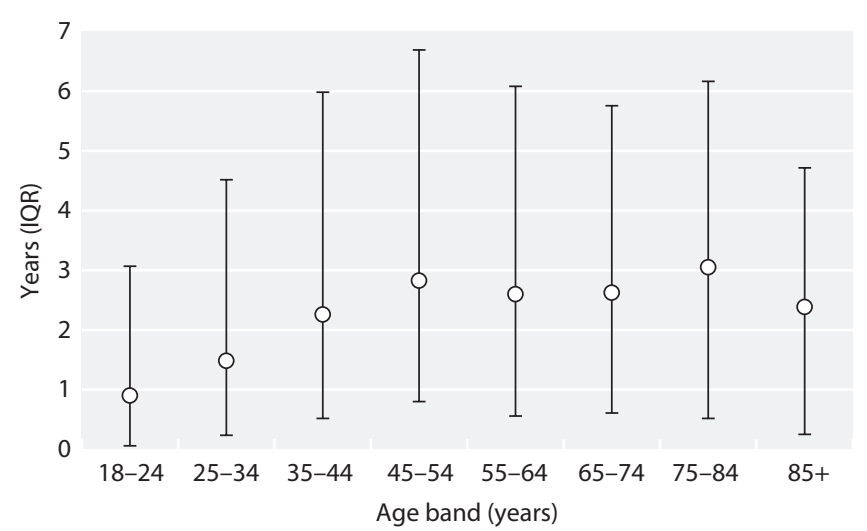

Fig. 1.13. Median duration of pre-RRT care by age group (incident patients 2012/2013)

\section{Ethnicity and late presentation}

In the 2012/2013 cohort, the percentage of South Asian and Black patients presenting late ( $<90$ days) was somewhat lower than in Whites $(16.3 \%$ vs. $18.7 \%$ : $p=0.02)$.

\section{Primary renal disease and late presentation}

In the 2012/2013 cohort, late presentation differed significantly between primary renal diagnoses (Chisquared test $p<0.0001$ ) (table 1.17). Patients in the acute group or with data not available had high rates of late presentation as anticipated. Those with diabetes and pyelonephritis or adult polycystic kidney disease had low rates in keeping with their longer natural histories of $\mathrm{CKD}$ progression. There was a notable decline in the proportion of diabetics presenting late up until 2007. Since then the proportion has been stable. The decline seen earlier likely reflects national initiatives to screen patients with diabetes for proteinuria and falling GFR.

\section{Modality and late presentation}

In the 2012/2013 cohort, late presentation was associated with initial modality. The percentage of patients whose first modality was PD was significantly lower in the late presentation group than in those presenting earlier (10.8\% vs. $22.2 \%$ : $p<0.0001)$. By 90 days after

Table 1.17. Late presentation by primary renal diagnosis (2012/2013 incident patients)

\begin{tabular}{lrrr}
\hline & & \multicolumn{2}{c}{ Late presentation } \\
\cline { 3 - 4 } Diagnosis & $N$ & $N$ & $\%$ \\
\hline Uncertain aetiology & 1,365 & 284 & 20.8 \\
Diabetes & 2,473 & 234 & 9.5 \\
Glomerulonephritis & 1,317 & 196 & 14.9 \\
Other identified category & 1,092 & 195 & 17.9 \\
Polycystic kidney or & 1,366 & 132 & 9.7 \\
pyelonephritis & & & \\
Renal vascular disease & 1,198 & 196 & 16.4 \\
Acute group & 886 & 489 & 55.2 \\
Data not available & 264 & 85 & 32.2 \\
\hline Unilke elsewhere in the report, & & &
\end{tabular}

Unlike elsewhere in the report, the RVD group includes hypertension Polycystic and pyelonephritis are grouped together

Acute group includes crescentic (extracapillary) glomerulonephritis (type I, II, III), nephropathy (interstitial) due to cis-platinum, renal vascular disease due to malignant hypertension, renal vascular disease due to polyarteritis, Wegener's granulomatosis, cryoglobulinemic glomerulonephritis, myelomatosis/light chain deposit disease, Goodpasture's syndrome, systemic sclerosis (scleroderma), haemolytic ureaemic syndrome, multi-system disease - other, tubular necrosis (irreversible) or cortical necrosis, Balkan nephropathy, kidney tumour(s), and traumatic or surgical loss of kidney(s) 
Table 1.18. Percentage prevalence of specific comorbidities amongst patients presenting late $(<90$ days $)$ compared with those presenting early ( $\geqslant 90$ days) $(2012 / 2013$ incident patients)

\begin{tabular}{lccc}
\hline Comorbidity & $<90$ days & $\geqslant 90$ days & $p$-value \\
\hline Ischaemic heart disease & 15.9 & 19.7 & 0.003 \\
Cerebrovascular disease & 9.4 & 10.6 & 0.2 \\
Peripheral vascular disease & 10.4 & 12.0 & 0.1 \\
Diabetes (not a cause of ERF) & 9.2 & 9.9 & 0.5 \\
Liver disease & 4.3 & 2.8 & 0.01 \\
Malignancy & 21.3 & 11.5 & $<0.0001$ \\
COPD & 7.7 & 7.3 & 0.6 \\
Smoking & 15.2 & 13.6 & 0.2 \\
\hline
\end{tabular}

RRT initiation this difference was reduced, although it was still highly significant ( $12.8 \%$ vs. $22.0 \%$ : $p<0.0001)$.

\section{Comorbidity and late presentation}

In the 2012/2013 cohort, the percentage of patients who were assessed as having no comorbidity was similar in those who presented late as in those presenting earlier ( $45.2 \%$ vs. $47.4 \%: p=0.2$ ). That said however, there were differences in those with comorbidities: ischaemic heart disease was significantly less common and liver disease and malignancy significantly more common in those presenting late compared to those presenting early (table 1.18) perhaps reflecting underlying causes of $\mathrm{CKD}$ and its progression. This is in keeping with findings from other studies [6-8].

\section{Haemoglobin and late presentation}

In the 2012/2013 cohort, patients presenting late had a significantly lower average haemoglobin concentration at RRT initiation than patients presenting earlier (91 vs.
$101 \mathrm{~g} / \mathrm{L}: p<0.0001)$. This may reflect inadequate predialysis care with limited anaemia management, but alternatively those presenting late may be more likely to have anaemia because of multisystem disease or intercurrent illness. More detailed analyses of haemoglobin at start of RRT and late presentation can be found in chapter 7: Haemoglobin, Ferritin and Erythropoietin amongst UK Adult Dialysis Patients in 2013.

\section{eGFR at start of RRT and late presentation}

In the 2012/2013 cohort, eGFR at start of RRT was significantly lower in patients presenting late than those presenting earlier ( 7.7 vs. $8.6 \mathrm{ml} / \mathrm{min} / 1.73 \mathrm{~m}^{2}: p<0.0001$ ). Although these findings are in contrast to some of the studies in the literature, many of those studies pre-date the era of routine use of eGFR [6, 7]. A recent Cochrane review has shown that eGFR was indeed higher in RRT patients [9] referred early (mean difference of $0.42 \mathrm{ml} /$ $\min / 1.73 \mathrm{~m}^{2}$ ) compared to those presenting late (definition: up to 6 months before starting RRT) consistent with UKRR data.

\section{International comparisons}

Figure 1.14 shows the crude RRT incidence rates (including children) for 2011 for several countries. The data is from the USRDS [10]; 2011 was the latest year available at time of writing. The UK incidence rate was similar to those in many other Northern European countries, Australia and New Zealand but remained markedly lower than in some other countries, most

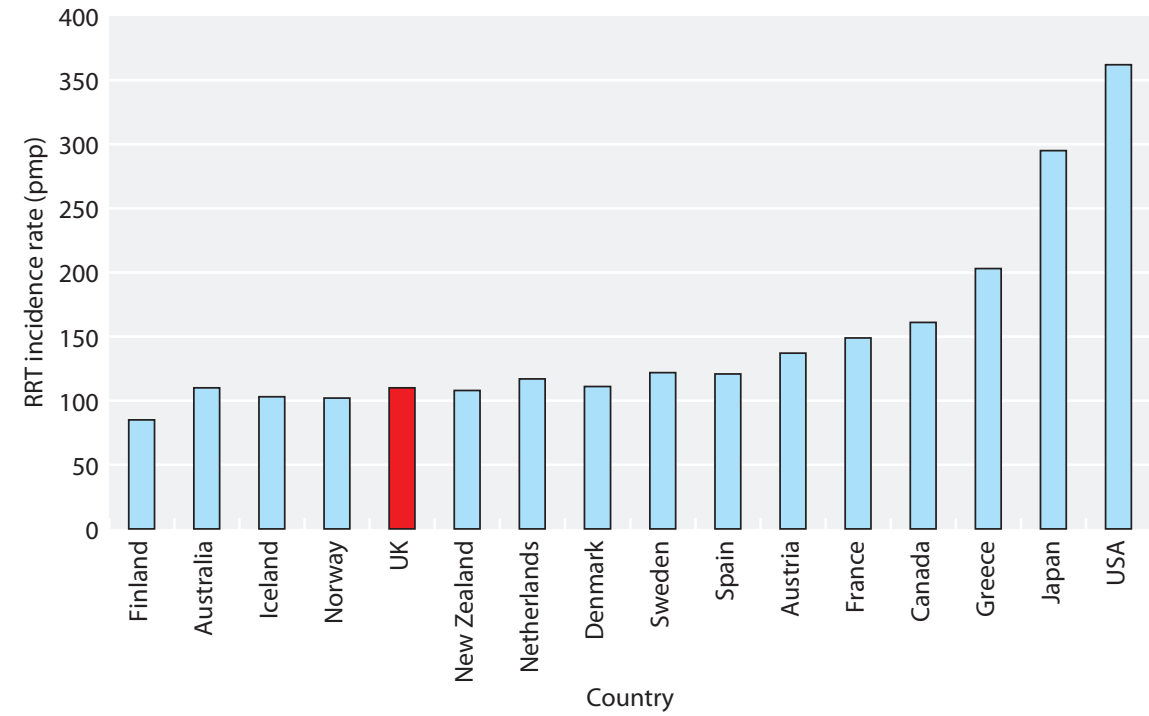

28
Fig. 1.14. International comparison of RRT incidence rates in 2011 Non UK data from USRDS [10] 
notably Greece, Japan and the USA. There are numerous reasons for these differences which have been documented and explored in other ecological studies and summarised by this review [11].

\section{Survival of incident patients}

See chapter 5: Survival and Causes of Death of UK Adult Patients on Renal Replacement Therapy in 2013.

\section{Conclusions}

Across the UK, as a whole, the renal replacement therapy (RRT) incidence rate for 2013 was similar to those in 2012 and 2011. Partly because of the smaller numbers involved, rates have been more variable over the last few years for Northern Ireland, Scotland and Wales compared with England. Wales continues to have the highest incidence rate and there remains large between centre variation in incidence rates for RRT some of which is likely explained by population differences in ethnicity and age structure. There was a seven-fold variation between CCG/HBs in the rates of older people $(>75)$ starting RRT and also substantial between centre variation in use of different types of RRT modality some of which suggests inefficient use of cheaper and more effective forms of treatment. Although significant numbers of patients continue to present late to renal centres this proportion has dropped substantially in the last eight years. Some centre's lower rates $(<10 \%)$ suggest that local factors may be worth exploring in improving this aspect of renal care. Plans for more frequent and more detailed data downloads will hopefully allow the UKRR to explore these areas of variation in advanced CKD care.

Conflicts of interest: none

\section{References}

$1 \mathrm{http} / / /$ www.renal.org/guidelines/modules/planning-initiating-and-withdrawal-of-renal-replacement-therapy\#sthash.IDPHwzZI.dpbs

-2 Levey AS, Coresh J, Greene T, Stevens LA, Zhang YL, Hendriksen S, Kusek JW, Van Lente F: Chronic Kidney Disease Epidemiology Collaboration. Using standardized serum creatinine values in the modification of diet in renal disease study equation for estimating glomerular filtration rate. Ann Intern Med. 2006;145(4):247-54

3 http://www.nomisweb.co.uk/census/2011/LC2101EW/view/2092957703? rows $=\mathrm{c}$ ethpuk $11 \& \mathrm{cols}=\mathrm{c}$ age

4 Ford DJ, Fogarty DG, Steenkamp R, Tomson CRV, Ben-Shlomo Y, Ansell D: Chapter 13: The UK Renal Registry Advanced CKD Study: frequency of incorrect reporting of date of start of RRT. Nephron Clinical Practice;115(suppl 1):c271-c78

$5 \mathrm{http} / / /$ www.renal.org/CKDguide/full/UKCKDfull.pdf

-6 Kazmi WH et al.: Late nephrology referral and mortality among patients with end-stage renal disease: a propensity score analysis. Nephrology Dialysis Transplantation, 2004;19(7):1808-1814
7 Roubicek C, et al.: Timing of nephrology referral: Influence on mortality and morbidity. American journal of kidney diseases: the official journal of the National Kidney Foundation, 2000;36(1):35-41

8 Winkelmayer WC, et al.: A Propensity Analysis of Late Versus Early Nephrologist Referral and Mortality on Dialysis. Journal of the American Society of Nephrology, 2003;14(2):486-492

-9 Cochrane Database Syst Rev: Early referral to specialist nephrology services for preventing the progression to end-stage kidney disease. 2014 Jun 18;6:CD007333. doi: 10.1002/14651858.CD007333.pub2

10 US Renal Data System, USRDS 2013 Annual Data Report: Atlas of Chronic Kidney Disease and End-Stage Renal Disease in the United States, National Institutes of Health, National Institute of Diabetes and Digestive and Kidney Diseases, Bethesda, MD, 2013

$\checkmark 11$ Caskey FJ, Jager KJ: A population approach to renal replacement therapy epidemiology: lessons from the EVEREST study. Nephrol Dial Transplant. 2014 Aug;29(8):1494-9. doi: 10.1093/ndt/gft390. Epub 2013 Oct 28 